\title{
LOCAL EXISTENCE OF CLASSICAL SOLUTIONS TO THE TWO-DIMENSIONAL VISCOUS COMPRESSIBLE FLOWS WITH VACUUM*
}

\author{
ZHEN $\mathrm{LUO}^{\dagger}$
}

\begin{abstract}
We consider the Cauchy problem of the barotropic compressible Navier-Stokes equations in $2 \mathrm{D}$. The local existence of unique classical solution is established. We present some new elaborate estimates to bound the $L^{p}$-norm of the velocity $u$. The far field density is constant state, which could be either vacuum or non-vacuum. The initial density is allowed to vanish and the spatial measure of the set of vacuum can be arbitrarily large.
\end{abstract}

Key words. Compressible Navier-Stokes, vacuum, Cauchy problem, local existence.

AMS subject classifications. 35B45, 35M10, 35Q30, 76N10.

\section{Introduction}

The motion of a compressible, viscous, barotropic Newtonian fluid occupying a domain $\Omega \subset \mathbb{R}^{N}$ is governed by the compressible Navier-Stokes equations:

$$
\left\{\begin{array}{l}
\rho_{t}+\operatorname{div}(\rho u)=0, \\
(\rho u)_{t}+\operatorname{div}(\rho u \otimes u)-\mu \Delta u-(\mu+\lambda) \nabla(\operatorname{div} u)+\nabla P(\rho)=0,
\end{array}\right.
$$

where $\rho \geq 0, u=\left(u^{1}, \cdots, u^{N}\right)$, and $P=a \rho^{\gamma}(a>0, \gamma>1)$ are the fluid density, velocity, and pressure, respectively. The constant viscosity coefficients $\mu$ and $\lambda$ satisfy the physical restrictions

$$
\mu>0, \quad 2 \mu+N \lambda \geq 0 .
$$

Let $\Omega=\mathbb{R}^{2}$ and $\tilde{\rho}$ be a fixed nonnegative constant. We look for the solutions $(\rho(x, t), u(x, t))$ to the Cauchy problem for (1.1) with the far field behavior

$$
u(x, t) \rightarrow 0, \quad \rho(x, t) \rightarrow \tilde{\rho} \geq 0, \quad \text { as }|x| \rightarrow \infty,
$$

and initial data,

$$
\left.(\rho, u)\right|_{t=0}=\left(\rho_{0}, u_{0}\right), \quad x \in \mathbb{R}^{2} .
$$

There is a vast literature studying the fluid dynamics. In particular, for (1.1), the one-dimensional problem has been studied extensively by many mathematicians; see Kazhikhov-Shelukhin [15] for sufficient smooth data, Serre [21,22] and Hoff [10] for discontinuous initial data, and the references therein. For the multi-dimensional case, the local existence and uniqueness of classical solutions without vacuum are known by Nash [19] and Serrin [23], respectively. Matsumura and Nishida showed in their pioneering paper [18] that the classical solutions exist globally in time for initial data close to a non vacuum equilibrium. For the case that the initial density is allowed

\footnotetext{
*Received: June 16, 2011; accepted (in revised version): July 30, 2011. Communicated by Zhouping Xin.

†School of Mathematical Sciences, Xiamen University, Xiamen, 361005, P.R. China (lluozhen@ gmail.com). This research is supported in part by NSFC (Grant Nos.10801111, 11171223), the Fundamental Research Funds for the Central Universities (Grant No.2010121006), and the Natural Science Foundation of Fujian Province of P.R. China (Grant No.2010J05011).
} 
to vanish, in fundamental work [16] Lions developed an existence theory of global in time weak solutions (see also Feireisl [8]). Better regularity of spatially periodic weak solutions in both two and three spatial dimensions was proved by Desjardins [7] for small time. Later, Hoff [12] obtained a new type of global weak solutions which has extra regularity information if the far field density is away from vacuum. Note that in [12] the weak solutions may contain vacuum even though the spatial measure of the set of vacuum is small. Recently, for dimension $N \geq 3$, the local existence and uniqueness of classical solutions were obtained by Cho, Choe, Kim [3, 4,6] and SalviStraškraba [20] for the case that the initial density need not be positive and may vanish in open sets. Later on, Huang-Li-Xin [14] showed that classical solutions exist globally in time with smooth initial data which is of small energy but possibly large oscillations in $\mathbb{R}^{3}$, that is, possibly with initial vacuum.

However, the existence of strong or classical solutions to the two-dimensional (2D) Cauchy problem for (1.1) is still open, especially when the initial density has compact support. In this paper, we will investigate the local existence of classical solutions to the Cauchy problem for the barotropic compressible Navier-Stokes equations (1.1) in $2 \mathrm{D}$ with constant far field state which could be either vacuum or non-vacuum. The initial density is allowed to vanish and the spatial measure of the set of vacuum can be arbitrarily large, in particular, the initial density can even have compact support.

We now give a brief overview of the analysis in this paper. We start with the basic energy estimate and succeed in deriving estimates on the $L^{2}$-norm of the gradient and the material derivatives of the velocity. This is achieved by modifying the basic estimates on the material derivatives of the velocity developed by Hoff [11]. Next, some new elaborate estimates are needed to overcome the difficulties, which in fact are the main ones of this paper, in bounding the $L^{p}$-norm of the velocity $u$ and the material derivatives of the velocity, $\dot{u}$. Combining some careful estimates on suitable spatially weighted norms of $\nabla u$ and $\nabla \dot{u}$ with the Caffarelli-Kohn-Nirenberg inequality, Lemma 2.1, we obtain the desired bounds on the $L^{p}$-norms of $u$ and $\dot{u}$ for some constant $p=p(\mu, \lambda)$. These facts, together with the Schauder fixed point theorem, Lemma 2.3, are enough to close our arguments.

Before stating the main result, we explain the notations and conventions used throughout this paper. For $\Omega \subset \mathbb{R}^{2}$, we denote

$$
\int f d x=\int_{\Omega} f d x
$$

For $1 \leq r \leq \infty, k \in \mathbb{N}$ we denote the standard homogeneous and inhomogeneous Sobolev spaces as follows:

$$
\left\{\begin{array}{l}
L^{r}=L^{r}(\Omega), \quad D^{k, r}=\left\{u \in L_{l o c}^{1}(\Omega) \mid\left\|\nabla^{k} u\right\|_{L^{r}}<\infty\right\}, \quad\|u\|_{D^{k, r}} \triangleq\left\|\nabla^{k} u\right\|_{L^{r}}, \\
W^{k, r}=L^{r} \cap D^{k, r}, \quad H^{k}=W^{k, 2}, \quad D^{k}=D^{k, 2}, \quad D_{0}^{k}=\left\{u \in D^{k}|u|_{\partial \Omega}=0\right\} .
\end{array}\right.
$$

Then our main result in this paper can be stated as follows:

TheOREM 1.1. For $\tilde{\rho} \geq 0$ and $\Omega=\mathbb{R}^{2}$, assume that the initial data $\left(\rho_{0} \geq 0, u_{0}\right)$ satisfy

$$
u_{0} \in D^{1} \cap D^{3}, \quad\left(\rho_{0}-\tilde{\rho}, P\left(\rho_{0}\right)-P(\tilde{\rho})\right) \in H^{3},
$$

and the compatibility condition

$$
-\mu \triangle u_{0}-(\mu+\lambda) \nabla \operatorname{divu}_{0}+\nabla P\left(\rho_{0}\right)=\rho_{0} g
$$


for some $g \in D^{1}$ with $\rho_{0}^{1 / 2} g \in L^{2}$. Moreover, if $\tilde{\rho}=0$, in addition to (1.5) and (1.6), suppose that

$$
|x|^{\alpha / 2} \nabla u_{0} \in L^{2}, \quad|x|^{\alpha / 2} P\left(\rho_{0}\right) \in L^{2}, \quad|x|^{\alpha / 2} \rho_{0}^{1 / 2} g \in L^{2},
$$

where

$$
\alpha \triangleq \mu /(4(2 \mu+\lambda)) \in(0,1 / 8]
$$

Then there exist a small time $T^{*}>0$ and a unique strong solution $(\rho, u)$ to the Cauchy problem (1.1), (1.3), (1.4) on $\mathbb{R}^{2} \times\left[0, T^{*}\right]$ such that for any $\tau \in\left(0, T^{*}\right)$,

$$
\left\{\begin{array}{l}
(\rho-\tilde{\rho}, P-P(\tilde{\rho})) \in C\left(\left[0, T^{*}\right] ; H^{3}\right), \\
u \in C\left(\left[0, T^{*}\right] ; L^{q} \cap D^{1} \cap D^{3}\right) \cap L^{2}\left(0, T^{*} ; D^{4}\right) \cap L^{\infty}\left(\tau, T^{*} ; D^{4}\right), \\
u_{t} \in L^{\infty}\left(0, T^{*} ; D^{1}\right) \cap L^{2}\left(0, T^{*} ; L^{q} \cap D^{2}\right) \\
u_{t} \in L^{\infty}\left(\tau, T^{*} ; L^{q_{1}} \cap D^{2}\right) \cap H^{1}\left(\tau, T^{*} ; D^{1}\right) \\
\sqrt{\rho} u_{t} \in L^{\infty}\left(0, T^{*} ; L^{2}\right), \quad \sqrt{\rho} u_{t t} \in L^{2}\left(0, T^{*} ; L^{2}\right), \\
\sqrt{\rho} u_{t t} \in L^{\infty}\left(\tau, T^{*} ; L^{2}\right),
\end{array}\right.
$$

where

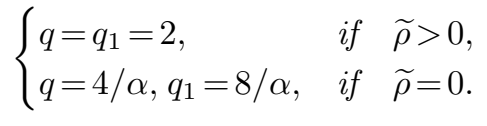

A few remarks are in order:

REMARK 1.2. The smoothing effect of the velocity $u$ in $t \in\left[\tau, T^{*}\right], \forall \tau \in\left(0, T^{*}\right)$ takes us from the strong solution obtained in Theorem 1.1 to a classical one in $\mathbb{R}^{2} \times\left(0, T^{* *}\right]$, for some $T^{* *} \in\left(0, T^{*}\right]$ (see [4]).

Remark 1.3. The a priori estimates in $[3,4,6,20]$ for the three-dimensional case cannot be applied here. The main reason is that the $2 \mathrm{D}$ case is the critical case for the standard Sobolev embedding theorem and it seems difficult to bound the $L^{p}$-norm of the velocity just in terms of the $L^{2}$-norm of the gradient of it.

REMARK 1.4. According to the blow up phenomena obtained in [13], the $C^{1}\left([0, T] ; H^{s}\right)(s>2)$ solution to the $2 \mathrm{D}$ spherically symmetric compressible NavierStokes Equations (1.1) with compactly supported initial density must blow up in finite time. Therefore, for 2D compressible Navier-Stokes equations, the solutions in our main result, Theorem 1.1, give a kind of class of solutions which are expected to exist globally in time in the case that $\tilde{\rho}=0$.

The rest of the paper is organized as follows: In Section 2, we recall some known results and inequalities which will be needed in later analysis. Section 3 is devoted to deriving the necessary a priori estimates on classical solutions for $\tilde{\rho}=0$. Finally, the main result, Theorem 1.1, is proved in Section 4.

\section{Preliminaries}

First, the following Caffarelli-Kohn-Nirenberg inequality will play a key role in obtaining the $L^{q}$ estimate of $u$ when $\widetilde{\rho}=0$.

Lemma 2.1. ([1,2]) For $\alpha \in(0,2)$, the following estimates hold for all $u \in C_{0}^{\infty}\left(\mathbb{R}^{2}\right)$ :

$$
\int|x|^{\alpha-2}|u|^{2} d x \leq \frac{\alpha^{2}}{4} \int|x|^{\alpha}|\nabla u|^{2} d x, \quad\|u\|_{L^{4 / \alpha}}^{2} \leq C(\alpha) \int|x|^{\alpha}|\nabla u|^{2} d x .
$$


Next, we recall some known results which will be also used later.

Lemma 2.2. ([24] Aubin-Lions) Let $X_{0}, X$, and $X_{1}$ be three Banach spaces with $X_{0} \subset X \subset X_{1}$. Suppose that $X_{0}$ is compactly embedded in $X$ and that $X$ is continuously embedded in $X_{1}$.

i) Let $G$ be bounded in $L^{p}\left(0, T ; X_{0}\right)$ where $1 \leq p<\infty$, and $\partial G / \partial t$ be bounded in $L^{1}\left(0, T ; X_{1}\right)$. Then $G$ is relatively compact in $L^{p}(0, T ; X)$.

ii) Let $F$ be bounded in $L^{\infty}\left(0, T ; X_{0}\right)$ and $\partial F / \partial t$ be bounded in $L^{r}\left(0, T ; X_{1}\right)$ where $r>1$. Then $F$ is relatively compact in $C([0, T] ; X)$.

Lemma 2.3. ( [9] Schauder) Let $\Re$ be a compact convex set in a Banach space $\mathcal{B}$ and let $\mathcal{T}$ be a continuous mapping of $\Re$ into itself. Then $\mathcal{T}$ has a fixed point, that is, $\mathcal{T} x=x$ for some $x \in \Re$.

Lemma 2.4. ( [17] Gagliardo-Nirenberg) For $p \in[2, \infty), q \in(1, \infty)$, and $r \in(2, \infty)$, there exists some generic constant $C>0$ which may depend on $p, q, r$ such that for $f \in H^{1}\left(\mathbb{R}^{2}\right)$ and $g \in L^{q}\left(\mathbb{R}^{2}\right) \cap D^{1, r}\left(\mathbb{R}^{2}\right)$, we have

$$
\begin{gathered}
\|f\|_{L^{p}} \leq C\|f\|_{L^{2}}^{2 / p}\|\nabla f\|_{L^{2}}^{(p-2) / p}, \\
\|g\|_{C\left(\overline{\mathbb{R}^{2}}\right)} \leq C\|g\|_{L^{q}}^{q(r-2) /(2 r+q(r-2))}\|\nabla g\|_{L^{r}}^{2 r /(2 r+q(r-2))} .
\end{gathered}
$$

Finally, the following lemma will be used to bound the $L^{2}$-norm of $u$ when $\widetilde{\rho}>0$.

Lemma 2.5. If $\tilde{\rho}>0$, then there exists some constant $C(\tilde{\rho})>0$ such that the following estimate holds for $\rho-\tilde{\rho} \in L^{2}\left(\mathbb{R}^{2}\right), u \in D^{1}\left(\mathbb{R}^{2}\right), \rho^{1 / 2} u \in L^{2}\left(\mathbb{R}^{2}\right)$,

$$
\|u\|_{L^{2}\left(\mathbb{R}^{2}\right)}^{2} \leq C\left(\int_{\mathbb{R}^{2}} \rho|u|^{2} d x+\|\tilde{\rho}-\rho\|_{L^{2}\left(\mathbb{R}^{2}\right)}^{2}\|\nabla u\|_{L^{2}\left(\mathbb{R}^{2}\right)}^{2}\right) .
$$

Proof. (2.4) follows directly from the following simple fact that

$$
\begin{aligned}
\tilde{\rho} \int_{\mathbb{R}^{2}}|u|^{2} d x & =\int_{\mathbb{R}^{2}} \rho|u|^{2} d x+\int_{\mathbb{R}^{2}}(\tilde{\rho}-\rho)|u|^{2} d x \\
& \leq \int_{\mathbb{R}^{2}} \rho|u|^{2} d x+\|\tilde{\rho}-\rho\|_{L^{2}\left(\mathbb{R}^{2}\right)}\|u\|_{L^{4}\left(\mathbb{R}^{2}\right)}^{2} \\
& \leq \int \rho|u|^{2} d x+C\|\tilde{\rho}-\rho\|_{L^{2}\left(\mathbb{R}^{2}\right)}\|u\|_{L^{2}\left(\mathbb{R}^{2}\right)}\|\nabla u\|_{L^{2}\left(\mathbb{R}^{2}\right)} .
\end{aligned}
$$

3. A priori estimates for $\widetilde{\rho}=0$

In this section, for $\widetilde{\rho}=0$, we derive some uniform local (in time) a priori estimates on the solutions to the following linearized problems in a bounded smooth domain $\Omega \subset \mathbb{R}^{2}$. The estimates we obtain here are independent of the lower bound of $\rho_{0}$ and the size of the domain.

First, we consider the following linearized hyperbolic problem:

$$
\begin{cases}\rho_{t}+\operatorname{div}(\rho v)=0, & \text { in } \Omega \times(0, T), \\ \rho(x, 0)=\rho_{0}, & \text { in } \Omega,\end{cases}
$$


where $v$ is a known vector field in $\Omega \times(0, T)$ such that

$$
v \in C\left([0, T] ; H_{0}^{1} \cap H^{3}\right) \cap L^{2}\left(0, T ; H^{4}\right) .
$$

It is quite well-known that the linear problem (3.1) has a unique strong solution $\rho$ for any regular initial data $\rho_{0}$ because the vector field $v$ is sufficiently smooth. In fact, the following result is proved essentially in [5] (see [4] also), and thus stated without a detailed proof.

Lemma 3.1. Assume that (3.2) holds and that $0 \leq \rho_{0} \in H^{3}(\Omega)$. Then

(i) there exists a unique solution $\rho$ to the problem (3.1) such that

$$
\rho \in C\left([0, T] ; H^{3}\right), \quad \rho_{t} \in C\left([0, T] ; H^{2}\right) ;
$$

(ii) the solution $\rho$ satisfies the following estimate:

$$
\|\rho(t)\|_{H^{3}} \leq\left\|\rho_{0}\right\|_{H^{3}} \exp \left(C \int_{0}^{t}\|v(s)\|_{D^{1} \cap D^{4}} d s\right),
$$

for some universal constant $C$;

(iii) the solution $\rho$ is represented by the formula

$$
\rho(x, t)=\rho_{0}(U(0 ; x, t)) \exp \left\{-\int_{0}^{t} \operatorname{div} v(U(s ; x, t), s) d s\right\}
$$

where $U \in C([0, T] ;[0, T] \times \Omega)$ is the solution to the initial value problem

$$
\begin{cases}\frac{\partial}{\partial s} U(s ; x, t)=v(s ; U(s ; x, t)), & 0 \leq s \leq T \\ U(t ; x, t)=x, & 0 \leq s \leq T, x \in \bar{\Omega}\end{cases}
$$

Next, we deal with the following linearized parabolic problem:

$$
\begin{cases}\rho u_{t}+\rho v \cdot \nabla u-\mu \Delta u-(\mu+\lambda) \nabla(\operatorname{div} u)=-\nabla P(\rho), & \text { in } \Omega \times(0, T), \\ u=0, & \text { on } \partial \Omega \times(0, T), \\ u(x, 0)=u_{0}, & \text { in } \Omega,\end{cases}
$$

where $\rho$ is a known scalar field in $(0, T) \times \Omega$ such that

$$
\rho, \rho^{\gamma} \in C\left([0, T] ; H^{3}\right), \quad \rho_{t},\left(\rho^{\gamma}\right)_{t} \in C\left([0, T] ; H^{2}\right), \quad \rho \geq \delta \text { on } \Omega \times[0, T],
$$

for some constant $\delta>0$. Recall that $L \triangleq-\mu \Delta-(\mu+\lambda) \nabla$ div is a strongly elliptic operator (see [3] for instance). The following existence and regularity results on solutions to the linear parabolic problem (3.7) can be proved by applying a standard method such as a semidiscrete Galerkin method or the method of continuity (see $[5,7]$ ).

Lemma 3.2. (i) Assume that $u_{0} \in H_{0}^{1}$, (3.2), and (3.8) hold. Then there exists a unique strong solution $u$ to the problem (3.7) such that

$$
u \in C\left([0, T] ; H_{0}^{1}\right) \cap L^{2}\left(0, T ; H^{2}\right), u_{t} \in L^{2}\left(0, T ; L^{2}\right) .
$$

(ii) If in addition $u_{0} \in H_{0}^{1} \cap H^{2}$ and $v_{t} \in L^{\infty}\left(0, T ; L^{2}\right)$, then the solution $u$ satisfies

$$
u \in L^{\infty}\left(0, T ; H^{2}\right), u_{t} \in L^{2}\left(0, T ; H_{0}^{1}\right), u_{t t} \in L^{2}\left(0, T ; H^{-1}\right) .
$$


(iii) Finally, if in addition $u_{0} \in H_{0}^{1} \cap H^{3}, u_{t}(0)=\rho(0)^{-1}\left(\nabla P(\rho(0))-L u_{0}\right) \in H_{0}^{1}$, and $v_{t} \in L^{\infty}\left(0, T ; H_{0}^{1}\right)$, then the solution $u$ satisfies

$$
u \in L^{\infty}\left(0, T ; H^{3}\right), \quad u_{t} \in L^{2}\left(0, T ; H^{2}\right), \quad u_{t t} \in L^{2}\left(0, T ; L^{2}\right) .
$$

We now derive the local (in time) a priori estimates for $(\rho, u)$. These estimates are independent of the lower bound $\delta$ of $\rho_{0}$ and size of the domain $\Omega$. Set

$$
\begin{aligned}
c_{0} \triangleq & 1+\left\|\rho_{0}^{1 / 2} u_{0}\right\|_{L^{2}}^{2}+\left\|\left(\rho_{0}, P\left(\rho_{0}\right)\right)\right\|_{H^{3}}^{2}+\left\|u_{0}\right\|_{D^{1} \cap D^{3}}^{2} \\
& +\left\|\left(P\left(\rho_{0}\right),\left|\nabla u_{0}\right|, \rho_{0}^{1 / 2}|g|\right)\left(1+|x|^{\alpha / 2}\right)\right\|_{L^{2}}^{2}+\|g\|_{D^{1}}^{2} .
\end{aligned}
$$

For $\alpha$ as in (1.8), we define

$$
\Phi(v, t) \triangleq 1+\sup _{0 \leq s \leq t}\left(\|\nabla v\|_{H^{1}}^{2}+\left\||x|^{\alpha / 2} \nabla v\right\|_{L^{2}}^{2}\right)+\int_{0}^{t}\|\nabla v\|_{H^{1} \cap W^{1,4 / \alpha}}^{2} d s .
$$

From now on, we always assume that $0 \leq t \leq T \leq 1$ and $C$ will denote a generic positive constant depending on $\mu, \lambda, a, \gamma$.

We start off with the following energy estimate for $(\rho, u)$.

Lemma 3.3. Let $(\rho, u)$ be a smooth solution of (3.1), (3.7). Then

$$
\sup _{0 \leq s \leq t} \int\left(\rho|u|^{2}+P^{2}\right) d x+\int_{0}^{t} \int|\nabla u|^{2} d x d t \leq C c_{0} \exp \left\{\Phi(v, t) t^{1 / 2}\right\} .
$$

Proof. Note that $(3.1)_{1}$ implies that $P$ satisfies

$$
P_{t}+v \cdot \nabla P+\gamma P \operatorname{div} v=0 .
$$

It is easy to check that

$$
\left(\|(\rho, P)\|_{L^{2}}^{2}\right)_{t} \leq C\|\nabla v\|_{L^{\infty}}\|(\rho, P)\|_{L^{2}}^{2},
$$

which together with (3.5) yields that

$$
\begin{aligned}
& \sup _{0 \leq s \leq t}\left(\|(\rho, P)\|_{L^{2}}^{2}+\|(\rho, P)\|_{L^{\infty}}^{2}\right) \\
\leq & C c_{0} \exp \left\{\int_{0}^{t}\|\nabla v\|_{L^{\infty}} d s\right\} \\
\leq & C c_{0} \exp \left\{\Phi(v, t) t^{1 / 2}\right\} .
\end{aligned}
$$

Now, the energy estimate gives

$$
\frac{1}{2}\left(\int \rho|u|^{2} d x\right)_{t}+\int\left(\mu|\nabla u|^{2}+(\lambda+\mu)(\operatorname{div} u)^{2}\right) d x \leq C_{\delta}\|P\|_{L^{2}}^{2}+\delta\|\nabla u\|_{L^{2}}^{2},
$$

which together with (3.17) yields (3.14).

The following lemma will give preliminary $L^{2}$ bounds for $\nabla u$ and $\rho \dot{u}$ with $\dot{u} \triangleq$ $u_{t}+v \cdot \nabla u$. 
Lemma 3.4. Let $(\rho, u)$ be as in Lemma 3.3. Then

$$
\sup _{0 \leq s \leq t}\|\nabla u\|_{L^{2}}^{2}+\int_{0}^{t} \int \rho|\dot{u}|^{2} d x d t \leq C c_{0}^{2} \exp \left\{C c_{0} t \exp \{C \Phi(v, t)\}\right\},
$$

and

$$
\begin{aligned}
& B^{\prime}(t)+\int \rho|\dot{u}|^{2}|x|^{\alpha} d x \\
& \leq C\left(1+\|\nabla v\|_{L^{\infty}}+\|v\|_{L^{\infty}}\right)\left\||x|^{\alpha / 2} \nabla u\right\|_{L^{2}}^{2}+2 \mu \alpha^{4}\left\||x|^{\alpha / 2} \nabla \dot{u}\right\|_{L^{2}}^{2} \\
& \quad+C \beta c_{0}^{2} t \exp \{C \Phi(v, t)\},
\end{aligned}
$$

where

$$
\begin{aligned}
B(t) & \triangleq \int|x|^{\alpha}\left(\frac{\mu}{2}|\nabla u|^{2}+\frac{\mu+\lambda}{2}|\operatorname{div} u|^{2}-\operatorname{div} u P+\beta P^{2}\right) d x \\
& \geq \frac{\mu}{4} \int|x|^{\alpha}\left(|\nabla u|^{2}+P^{2}\right) d x
\end{aligned}
$$

for suitably large $\beta(\mu, \lambda)>0$.

Proof. Multiplying (3.7) by $\dot{u}$ then integrating the resulting equality over $\Omega$ leads to

$$
\begin{aligned}
\int \rho|\dot{u}|^{2} d x & =\int(-\dot{u} \cdot \nabla P+\mu \triangle u \cdot \dot{u}+(\lambda+\mu) \nabla \operatorname{div} u \cdot \dot{u}) d x \\
& \triangleq \sum_{i=1}^{3} M_{i} .
\end{aligned}
$$

Using (3.1) and integrating by parts gives

$$
\begin{aligned}
M_{1} & =-\int \dot{u} \cdot \nabla P d x \\
& =\int\left((\operatorname{div} u)_{t} P-(v \cdot \nabla u) \cdot \nabla P\right) d x \\
& =\left(\int \operatorname{div} u P d x\right)_{t}+\int\left(P^{\prime} \rho \operatorname{div} u \operatorname{div} v-P \operatorname{div} u \operatorname{div} v+P \partial_{i} v^{j} \partial_{j} u^{i}\right) d x \\
& \leq\left(\int \operatorname{div} u P d x\right)_{t}+C c_{0}^{1 / 2} \exp \left\{C \Phi(v, t) t^{1 / 2}\right\}\left(\|\nabla u\|_{L^{2}}^{2}+\|\nabla v\|_{L^{2}}^{2}\right),
\end{aligned}
$$

due to (3.17). Integration by parts implies

$$
\begin{aligned}
M_{2} & =\int \mu \triangle u \cdot \dot{u} d x \\
& =-\frac{\mu}{2}\left(\|\nabla u\|_{L^{2}}^{2}\right)_{t}-\mu \int \partial_{i} u^{j} \partial_{i}\left(v^{k} \partial_{k} u^{j}\right) d x \\
& \leq-\frac{\mu}{2}\left(\|\nabla u\|_{L^{2}}^{2}\right)_{t}+C\|\nabla v\|_{L^{\infty}}\|\nabla u\|_{L^{2}}^{2},
\end{aligned}
$$

and similarly,

$$
M_{3}=-\frac{\lambda+\mu}{2}\left(\|\operatorname{div} u\|_{L^{2}}^{2}\right)_{t}-(\lambda+\mu) \int \operatorname{div} u \operatorname{div}(v \cdot \nabla u) d x
$$




$$
\leq-\frac{\lambda+\mu}{2}\left(\|\operatorname{div} u\|_{L^{2}}^{2}\right)_{t}+C\|\nabla v\|_{L^{\infty}}\|\nabla u\|_{L^{2}}^{2} .
$$

Combining (3.16), (3.22)-(3.25) with Gronwall's inequality gives (3.19).

Next, multiplying (3.7) by $\dot{u}|x|^{\alpha}$, and then integrating the resulting equality over $\Omega$ leads to

$$
\begin{aligned}
\int \rho|\dot{u}|^{2}|x|^{\alpha} d x & =\int|x|^{\alpha}(-\dot{u} \cdot \nabla P+\mu \triangle u \cdot \dot{u}+(\lambda+\mu) \nabla \operatorname{div} u \cdot \dot{u}) d x \\
& \triangleq \sum_{i=1}^{3} M_{i}^{\prime} .
\end{aligned}
$$

Using (3.1) 1 and integrating by parts yields

$$
\begin{aligned}
M_{1}^{\prime}= & -\int|x|^{\alpha} \dot{u} \cdot \nabla P d x \\
= & \int\left(\left(|x|^{\alpha} \operatorname{div} u\right)_{t} P+\alpha x \cdot \dot{u}|x|^{\alpha-2} P+|x|^{\alpha} \operatorname{div}(v \cdot \nabla u) P\right) d x \\
= & \left(\int|x|^{\alpha} \operatorname{div} u P d x\right)_{t}+\alpha \int P|x|^{\alpha-2} x \cdot \dot{u} d x \\
& \quad+\int P|x|^{\alpha}\left((\gamma-1) \operatorname{div} u \operatorname{div} v+\partial_{i} v^{j} \partial_{j} u^{i}\right) d x \\
& \quad-\alpha \int|x|^{\alpha-2} P x \cdot(v \operatorname{div} u-v \cdot \nabla u) d x .
\end{aligned}
$$

Then,

$$
\begin{aligned}
M_{1}^{\prime} \leq & \left(\int|x|^{\alpha} \operatorname{div} u P d x\right)_{t}+\alpha\left(\int|x|^{\alpha-2}|\dot{u}|^{2} d x\right)^{1 / 2}\left(\int P^{2}|x|^{\alpha} d x\right)^{1 / 2} \\
& +C\|P\|_{L^{\infty}}\left\||x|^{\alpha / 2} \nabla v\right\|_{L^{2}}\left\||x|^{\alpha / 2} \nabla u\right\|_{L^{2}} \\
\leq & \left(\int|x|^{\alpha} \operatorname{div} u P d x\right)_{t}+\mu \alpha^{4} \int|x|^{\alpha}|\nabla \dot{u}|^{2} d x+C \int P^{2}|x|^{\alpha} d x \\
& +C\|P\|_{L^{\infty}}\left\||x|^{\alpha / 2} \nabla v\right\|_{L^{2}}^{2}+C\|P\|_{L^{\infty}}\left\||x|^{\alpha / 2} \nabla u\right\|_{L^{2}}^{2},
\end{aligned}
$$

where we have used (2.1). Integration by parts and (2.1) imply

$$
\begin{aligned}
M_{2}^{\prime}= & \mu \int|x|^{\alpha} \triangle u \cdot \dot{u} d x \\
=- & \frac{\mu}{2}\left(\left\||x|^{\alpha / 2} \nabla u\right\|_{L^{2}}^{2}\right)_{t}-\mu \int \partial_{i} u^{j}|x|^{\alpha} \partial_{i}\left(v^{k} \partial_{k} u^{j}\right) d x-\alpha \mu \int \partial_{i} u^{j} \dot{u}^{j}|x|^{\alpha-2} x_{i} d x \\
\leq- & \frac{\mu}{2}\left(\left\||x|^{\alpha / 2} \nabla u\right\|_{L^{2}}^{2}\right)_{t}+C\left(\|\nabla v\|_{L^{\infty}}+\|v\|_{L^{\infty}}\right)\left\|\nabla u|x|^{\alpha / 2}\right\|_{L^{2}}^{2} \\
& +C \alpha\left\|\nabla u|x|^{\alpha / 2}\right\|_{L^{2}}\left\|\dot{u}|x|^{\alpha / 2-1}\right\|_{L^{2}}+C\|v\|_{L^{\infty}}\|\nabla u\|_{L^{2}}^{2} \\
\leq- & \frac{\mu}{2}\left(\left\||x|^{\alpha / 2} \nabla u\right\|_{L^{2}}^{2}\right)_{t}+C\left(1+\|\nabla v\|_{L^{\infty}}+\|v\|_{L^{\infty}}\right)\left\||x|^{\alpha / 2} \nabla u\right\|_{L^{2}}^{2} \\
& +\frac{\mu}{2} \alpha^{4}\left\||x|^{\alpha / 2} \nabla \dot{u}\right\|_{L^{2}}^{2}+C\|v\|_{L^{\infty}}\|\nabla u\|_{L^{2}}^{2},
\end{aligned}
$$

and similarly,

$$
\begin{aligned}
M_{3}^{\prime} \leq & -\frac{\lambda+\mu}{2}\left(\left\||x|^{\alpha / 2} \operatorname{div} u\right\|_{L^{2}}^{2}\right)_{t}+C\left(1+\|\nabla v\|_{L^{\infty}}+\|v\|_{L^{\infty}}\right)\left\||x|^{\alpha / 2} \nabla u\right\|_{L^{2}}^{2} \\
& +\frac{\mu}{2} \alpha^{4}\left\||x|^{\alpha / 2} \nabla \dot{u}\right\|_{L^{2}}^{2}+C\|v\|_{L^{\infty}}\|\nabla u\|_{L^{2}}^{2} .
\end{aligned}
$$


Now, multiplying (3.15) by $P|x|^{\alpha}$, we obtain that

$$
\begin{aligned}
\left(\int|x|^{\alpha} P^{2} d x\right)_{t} & \leq C \int|x|^{\alpha-1} P^{2}|v| d x+C \int|x|^{\alpha} P^{2}|\operatorname{div} v| d x \\
& \leq C\|P\|_{L^{\infty}}\left(\int|x|^{\alpha} P^{2} d x\right)^{1 / 2}\left\||x|^{\alpha / 2} \nabla v\right\|_{L^{2}} \\
& \leq C c_{0}^{1 / 2} \exp \{C \Phi(v, t)\}\left(\int|x|^{\alpha} P^{2} d x\right)^{1 / 2}
\end{aligned}
$$

Then

$$
\sup _{0 \leq s \leq t} \int|x|^{\alpha} P^{2} d x \leq C c_{0} t \exp \{C \Phi(v, t)\},
$$

which together with (3.30) gives

$$
\left(\int|x|^{\alpha} P^{2} d x\right)_{t} \leq C c_{0}^{2} t \exp \{C \Phi(v, t)\} .
$$

Combining (3.26)-(3.29) and (3.32) implies (3.20).

Lemma 3.5. Let $(\rho, u)$ be as in Lemma 3.3. Then the following estimate holds for $q=4 / \alpha$ :

$$
\begin{aligned}
& \sup _{0 \leq s \leq t}(\left.\|\nabla(\rho, P)\|_{L^{2} \cap L^{q}}^{2}+\left\|\nabla^{2} u\right\|_{L^{2}}^{2}+\int\left(1+|x|^{\alpha}\right)\left(|\nabla u|^{2}+P^{2}+\rho|\dot{u}|^{2}\right) d x\right) \\
&+\int_{0}^{t} \int\left(1+|x|^{\alpha}\right)|\nabla \dot{u}|^{2} d x d t+\int_{0}^{t}\left\|\nabla^{2} u\right\|_{L^{q}}^{2} d t \\
& \leq C c_{0}^{3} \exp \left\{C c_{0} \exp \{C \Phi(v, t)\} t^{(q-2) /(2(q-1))}\right\} .
\end{aligned}
$$

Proof. First, applying $\partial_{t}+\partial_{k}\left(v^{k} \cdot\right)$ to Equation $(3.7)_{1}$ gives

$$
\begin{aligned}
& \quad \rho(\dot{u})_{t}+\rho v \cdot \nabla \dot{u} \\
& =\mu \Delta \dot{u}+(\mu+\lambda) \nabla \operatorname{div} \dot{u}-\mu \partial_{k}\left(\partial_{i} v^{k} \partial_{i} u\right)+\mu \partial_{i}\left(\partial_{k} v^{k} \partial_{i} u\right) \\
& \quad-\mu \partial_{i}\left(\partial_{i} v^{k} \partial_{k} u\right)+(\mu+\lambda)\left(\nabla(\operatorname{div} v \operatorname{div} u)-\nabla\left(\partial_{j} v^{k} \partial_{k} u^{j}\right)-\operatorname{div}\left(\nabla \mathrm{v}^{\perp} \operatorname{div} u\right)\right) \\
& \quad+\operatorname{div}\left(P \nabla v^{\perp}\right)+(\gamma-1) \nabla(P \operatorname{div} v) .
\end{aligned}
$$

After multiplying (3.34) by $\dot{u}$ and integrating over $\Omega$, in view of (3.19) and (3.14) we have

$$
\begin{aligned}
& \left(\int \rho|\dot{u}|^{2} d x\right)_{t}+\mu \int|\nabla \dot{u}|^{2} d x+(\mu+\lambda) \int|\operatorname{div} \dot{u}|^{2} d x \\
\leq & C \int|\nabla v|^{2}|\nabla u|^{2} d x+\int P^{2}|\nabla v|^{2} d x \\
\leq & C\|\nabla v\|_{L^{\infty}}^{2}\left(\|\nabla u\|_{L^{2}}^{2}+\|P\|_{L^{2}}^{2}\right) \\
\leq & C\|\nabla v\|_{L^{2}}^{(q-2) /(q-1)}\left\|\nabla^{2} v\right\|_{L^{q}}^{q /(q-1)}\left(\|\nabla u\|_{L^{2}}^{2}+\|P\|_{L^{2}}^{2}\right)
\end{aligned}
$$




$$
\leq C c_{0}^{2} \exp \left\{C c_{0} t^{1 / 2} \exp \{C \Phi(v, t)\}\right\}\|\nabla v\|_{L^{2}}^{(q-2) /(q-1)}\left\|\nabla^{2} v\right\|_{L^{q}}^{q /(q-1)},
$$

which yields

$$
\begin{aligned}
& \sup _{0 \leq s \leq t} \int \rho|\dot{u}|^{2} d x+\mu \int_{0}^{t} \int|\nabla \dot{u}|^{2} d x d t+(\mu+\lambda) \int_{0}^{t} \int|\operatorname{div} \dot{u}|^{2} d x d t \\
\leq & C c_{0}^{2}+C c_{0}^{2} \exp \left\{C c_{0} t^{1 / 2} \exp \{C \Phi(v, t)\}\right\} \Phi(v, t) t^{(q-2) /(2(q-1))} \\
\leq & C c_{0}^{2} \exp \left\{C c_{0} \exp \{C \Phi(v, t)\} t^{(q-2) /(2(q-1))}\right\}
\end{aligned}
$$

where $q=4 / \alpha$.

Next, multiplying (3.34) by $\dot{u}|x|^{\alpha}$ and integrating over $\Omega$ leads to

$$
\begin{aligned}
& \quad\left(\frac{1}{2} \int|x|^{\alpha} \rho|\dot{u}|^{2} d x\right)_{t}+\mu \int|x|^{\alpha}|\nabla \dot{u}|^{2} d x+(\mu+\lambda) \int|x|^{\alpha}|\operatorname{div} \dot{u}|^{2} d x \\
& \leq \alpha \int \rho|v||\dot{u}|^{2}|x|^{\alpha-1} d x+\mu \alpha \int|x|^{\alpha-1}|\nabla \dot{u}||\dot{u}| d x \\
& \quad+(\mu+\lambda) \alpha \int|x|^{\alpha-1}|\operatorname{div} \dot{u}||\dot{u}| d x \\
& \quad+C \int(|\nabla u||\nabla v|+P|\nabla v|)\left(|\nabla \dot{u}||x|^{\alpha}+\alpha|\dot{u}||x|^{\alpha-1}\right) d x \\
& \leq C \Phi^{1 / 2}(v, t) \int|x|^{\alpha} \rho|\dot{u}|^{2} d x+C\|v\|_{L^{\infty}} \int \rho|\dot{u}|^{2} d x+\alpha^{3}(2 \mu+\lambda) \int|x|^{\alpha}|\nabla \dot{u}|^{2} d x \\
& \quad+\delta \int|x|^{\alpha}|\nabla \dot{u}|^{2} d x+C_{\delta}\|\nabla v\|_{L^{\infty}}^{2} \int|x|^{\alpha}\left(|\nabla u|^{2}+P^{2}\right) d x
\end{aligned}
$$

Now from (1.8), we observe

$$
\begin{aligned}
& \left(\int|x|^{\alpha} \rho|\dot{u}|^{2} d x\right)_{t}+\mu \int|x|^{\alpha}|\nabla \dot{u}|^{2} d x+(\mu+\lambda) \int|x|^{\alpha}|\operatorname{div} \dot{u}|^{2} d x \\
& \leq C \Phi^{1 / 2}(v, t) \int|x|^{\alpha} \rho|\dot{u}|^{2} d x+C\|\nabla v\|_{L^{\infty}}^{2} B(t)+C\|v\|_{L^{\infty}} \int \rho|\dot{u}|^{2} d x .
\end{aligned}
$$

Recalling (3.20), we have

$$
\begin{aligned}
& \left(B(t)+\int|x|^{\alpha} \rho|\dot{u}|^{2} d x\right)_{t}+\frac{\mu}{2} \int|x|^{\alpha}|\nabla \dot{u}|^{2} d x \\
\leq & C \Phi^{1 / 2}(v, t) \int|x|^{\alpha} \rho|\dot{u}|^{2} d x+C c_{0}^{2} \exp \left\{C \exp \left\{C c_{0} \Phi(v, t)\right\} t^{\frac{q-2}{2(q-1)}}\right\} \Phi(v, t) \\
& +C\left(1+\|\nabla v\|_{L^{\infty}}+\|v\|_{L^{\infty}}+\|\nabla v\|_{L^{\infty}}^{2}\right) B(t) \\
\leq & C\left(\Phi(v, t)+\|\nabla v\|_{L^{2}}^{(q-2) /(q-1)}\left\|\nabla^{2} v\right\|_{L^{q}}^{q /(q-1)}\right)\left(B(t)+\int|x|^{\alpha} \rho|\dot{u}|^{2} d x\right) \\
& +C c_{0}^{2} \exp \left\{C c_{0} \exp \{C \Phi(v, t)\} t^{\frac{q-2}{2(q-1)}}\right\} \Phi(v, t), \quad \text { where } q=4 / \alpha .
\end{aligned}
$$

Thus, Gronwall's inequality implies 


$$
\begin{aligned}
& \sup _{0 \leq s \leq t}\left(B(t)+\int|x|^{\alpha} \rho|\dot{u}|^{2} d x\right) \\
\leq & C c_{0}^{2} \exp \left\{C \Phi(v, t) t+\Phi(v, t) t^{\frac{q-2}{2(q-1)}}\right\} \\
& +C c_{0}^{2} \exp \left\{C \Phi(v, t) t+\Phi(v, t) t^{\frac{q-2}{2(q-1)}}\right\} \exp \left\{C c_{0} \exp \{C \Phi(v, t)\} t^{\frac{q-2}{2(q-1)}}\right\} \Phi(v, t) t \\
\leq & C c_{0}^{2} \exp \left\{C c_{0} \exp \{C \Phi(v, t)\} t^{\frac{q-2}{2(q-1)}}\right\} .
\end{aligned}
$$

Combining (3.39) and (3.40), we discover

$$
\begin{aligned}
& \int_{0}^{t} \int|x|^{\alpha}|\nabla \dot{u}|^{2} d x d t \\
\leq & C c_{0}^{2}\left(\Phi(v, t) t+\Phi(v, t) t^{\frac{q-2}{2(q-1)}}\right) \exp \left\{C c_{0} \exp \{C \Phi(v, t)\} t^{\frac{q-2}{2(q-1)}}\right\} \\
& +C c_{0}^{2} \exp \left\{C c_{0} \exp \{C \Phi(v, t)\} t^{\frac{q-2}{2(q-1)}}\right\} \Phi(v, t) t \\
\leq & C c_{0}^{2} \exp \left\{C c_{0} \exp \{C \Phi(v, t)\} t^{\frac{q-2}{2(q-1)}}\right\} .
\end{aligned}
$$

Moreover, by virtue of (3.15), we have for $p \in[2,+\infty)$,

$$
\left(\|\nabla P\|_{L^{p}}\right)^{\prime} \leq C\|\nabla v\|_{L^{\infty}}\|\nabla P\|_{L^{p}}+C\|P\|_{L^{\infty}}\left\|\nabla^{2} v\right\|_{L^{p}},
$$

which together with (3.17) yields that for $p \in[2, q]$,

$$
\begin{aligned}
& \sup _{0 \leq s \leq t}\|\nabla P\|_{L^{p}} \\
\leq & C \exp \left\{C \int_{0}^{t}\|\nabla v\|_{L^{\infty}} d s\right\}\left(\left\|\nabla P\left(\rho_{0}\right)\right\|_{L^{p}}+\int_{0}^{t}\|P\|_{L^{\infty}}\left\|\nabla^{2} v\right\|_{L^{p}} d s\right) \\
\leq & C c_{0} \exp \left\{C \Phi(v, t) t^{1 / 2}\right\} .
\end{aligned}
$$

Similarly, we deduce from $(3.1)_{1}$ and (3.17) that

$$
\sup _{0 \leq s \leq t}\|\nabla \rho\|_{L^{p}} \leq C c_{0} \exp \left\{C \Phi(v, t) t^{1 / 2}\right\} .
$$

Note that $u$ satisfies

$$
\begin{cases}\mu \Delta u+(\mu+\lambda) \nabla \operatorname{div} u=\rho \dot{u}+\nabla P, & \text { in } \Omega, \\ u=0, & \text { on } \partial \Omega .\end{cases}
$$

Thus, we derive from a standard $L^{p}$ estimate for elliptic equations (see [9]) that for $p \in(1, \infty)$,

$$
\left\|\nabla^{2} u\right\|_{L^{p}} \leq C\left(\|\rho \dot{u}\|_{L^{p}}+\|\nabla P\|_{L^{p}}\right) .
$$

In view of (3.46), (3.41), (3.17), and (2.1), we have

$$
\int_{0}^{t}\left\|\nabla^{2} u\right\|_{L^{q}}^{2} d t
$$




$$
\begin{aligned}
& \leq C \int_{0}^{t}\left(\|\rho \dot{u}\|_{L^{q}}^{2}+\|\nabla P\|_{L^{q}}^{2}\right) d t \\
& \leq C \int_{0}^{t}\left(\|\rho\|_{L^{\infty}}^{2} \int|x|^{\alpha}|\nabla \dot{u}|^{2} d x+\|\nabla P\|_{L^{q}}^{2}\right) d t \\
& \leq C c_{0}^{3} \exp \left\{C c_{0} \exp \{C \Phi(v, t)\} t^{(q-2) /(2(q-1))}\right\} .
\end{aligned}
$$

Finally, it follows from (3.46), (3.36), and (3.17) that

$$
\sup _{0 \leq s \leq t}\left\|\nabla^{2} u\right\|_{L^{2}}^{2} \leq C c_{0}^{3} \exp \left\{C c_{0} \exp \{C \Phi(v, t)\} t^{(q-2) /(2(q-1))}\right\} .
$$

We finally present the following lemma to close our arguments on the a priori estimates.

Lemma 3.6. Assume that $(\rho, u)$ is as in Lemma 3.3. Then there exists a time $T^{*} \in$ $(0,1]$ depending only on $c_{0}, \mu, \lambda, a, \gamma$ such that

$$
\Phi\left(u, T^{*}\right) \leq M,
$$

provided $\Phi\left(v, T^{*}\right) \leq M$ with some given $M=M\left(\mu, \lambda, a, \gamma, c_{0}\right)>1$.

Proof. Lemmas 3.3-3.5 imply that for $q=4 / \alpha$,

$$
\Phi(u, t) \leq C c_{0}^{3} \exp \left\{C c_{0} \exp \{C \Phi(v, t)\} t^{(q-2) /(2(q-1))}\right\},
$$

which yields that

$$
\Phi\left(u, T^{*}\right) \leq M,
$$

by choosing $M=C c_{0}^{3} e^{C c_{0}}$ and

$$
T^{*}=\min \left\{e^{-2 C M(q-1) /(q-2)}, 1\right\} .
$$

\section{Proof of Theorem 1.1}

In this section, we will complete the proof of Theorem 1.1. We first use the Schauder fixed point theorem, Lemma 2.3, to obtain the following local existence of strong solutions in a bounded smooth domain for $\tilde{\rho}=0$.

Lemma 4.1. For $\widetilde{\rho}=0$ and a bounded smooth domain $\Omega \subset \mathbb{R}^{2}$, suppose that $\left(\rho_{0}, u_{0}\right)$ satisfies $\rho_{0} \geq \delta$ for some $\delta>0$ in addition to (1.5)-(1.7). Then for $T^{*}$ as in Lemma 3.6, there exists a unique strong solution $(\rho, u)$ to the problem (1.1) on $\Omega \times\left[0, T^{*}\right]$, with

$$
\left.(\rho, u)\right|_{t=0}=\left(\rho_{0}, u_{0}\right),\left.\quad u\right|_{\partial \Omega}=0
$$

satisfying

$$
\left\{\begin{array}{l}
(\rho, P) \in L^{\infty}\left(0, T^{*} ; W^{1, p}\right), \quad(\rho, P)_{t} \in L^{\infty}\left(0, T^{*} ; L^{p}\right), \\
u \in C\left(\left[0, T^{*}\right] ; L^{q} \cap D^{1} \cap D^{2}\right) \cap L^{2}\left(0, T^{*} ; D^{2, q}\right), \\
u_{t} \in L^{2}\left(0, T^{*} ; L^{q} \cap D^{1}\right), \quad \sqrt{\rho} u_{t} \in L^{\infty}\left(0, T^{*} ; L^{2}\right),
\end{array}\right.
$$


for $q=4 / \alpha$ and any $p \in[2, q]$. Moreover, the following estimate holds:

$$
\begin{aligned}
& \sup _{0 \leq t \leq T^{*}}\left(\int_{\Omega} \rho|u|^{2} d x+\int_{\Omega}\left(1+|x|^{\alpha}\right)\left(|\nabla u|^{2}+P^{2}+\rho|\dot{u}|^{2}\right) d x\right) \\
& \quad+\sup _{0 \leq t \leq T^{*}}\left(\left\|\nabla^{2} u\right\|_{L^{2}(\Omega)}+\|(\rho, P)\|_{W^{1, p}(\Omega)}+\left\|(\rho, P)_{t}\right\|_{L^{p}(\Omega)}\right) \\
& \quad+\int_{0}^{T^{*}} \int_{\Omega}\left(1+|x|^{\alpha}\right)|\nabla \dot{u}|^{2} d x d t+\int_{0}^{T^{*}}\left\|\nabla^{2} u\right\|_{L^{q}(\Omega)}^{2} d t \\
& \leq C\left(\mu, \lambda, a, \gamma, c_{0}\right) .
\end{aligned}
$$

Proof. For $T^{*}$ and $M$ as in Lemma 3.6, set

$$
\mathcal{B} \triangleq L^{2}\left(0, T^{*} ; H_{0}^{1}\right)
$$

and

$$
\Re \triangleq\left\{v \in L^{\infty}\left(0, T^{*} ; H_{0}^{1} \cap D^{2}\right) \cap L^{2}\left(0, T^{*} ; D^{2, q}\right) \mid v_{t} \in \mathcal{B}, \Phi\left(v, T^{*}\right) \leq M\right\} .
$$

It is easy to see from Lemma 2.2 that $\Re$ is a convex and compact subset of the Banach space $\mathcal{B}$. For any $v \in \Re$, by virtue of Lemma 3.1, there exists a unique solution $\rho=\rho(v)$ solving the Equation (3.1) on $\Omega \times\left[0, T^{*}\right]$ and satisfying (3.3). Moreover, according to Lemma 3.2, there exists a unique solution $u=\mathcal{T}(v, \rho(v))$ solving the problem (3.7) on $\Omega \times\left[0, T^{*}\right]$. Here, $(\rho, u)$ satisfies the a priori estimates stated in Lemmas 3.3-3.6, so that $\mathcal{T}$ maps $\Re$ to $\Re$.

Next, we will show $\mathcal{T}$ is a continuous operator in $\mathcal{B}$. By (3.33) and $(3.1)_{1}$, we have

$$
\sup _{0 \leq t \leq T^{*}}\left(\|\rho(v)\|_{W^{1, q}}+\left\|(\rho(v))_{t}\right\|_{L^{q}}\right) \leq C\left(\mu, \lambda, a, \gamma, c_{0}\right) .
$$

Let $v_{n} \in \Re, n=1,2, \cdots$, converge to $v$ in $\mathcal{B}$, that is,

$$
v_{n} \rightarrow v \quad \text { in } L^{2}\left(0, T^{*} ; H_{0}^{1}\right), \text { as } n \rightarrow+\infty,
$$

which together with $v_{n} \in \Re$ implies

$$
v_{n} \rightarrow v \quad w^{*} \text { in } L^{\infty}\left(0, T^{*} ; H_{0}^{1} \cap D^{2}\right) \cap L^{2}\left(0, T^{*} ; D^{2, q}\right) \text {, as } n \rightarrow+\infty .
$$

Thus, it follows from Lemma 2.2 and (4.4) that, up to a subsequence,

$$
\rho\left(v_{n_{j}}\right) \rightarrow \rho \quad \text { in } C\left(\bar{\Omega} \times\left[0, T^{*}\right]\right), \text { as } n_{j} \rightarrow+\infty .
$$

Taking the limits in (3.1) where $\rho, v$ are replaced by $\rho\left(v_{n_{j}}\right)$ and $v_{n_{j}}$ respectively, we obtain that $\rho$ is a weak solution to (3.1). Then, by the uniqueness of the weak solutions due to (4.6) and (4.7), we have $\rho=\rho(v)$, which again together with (4.7) and (4.4) implies

$$
\rho\left(v_{n}\right) \rightarrow \rho \quad \text { in } C\left(\bar{\Omega} \times\left[0, T^{*}\right]\right), \text { as } n \rightarrow+\infty,
$$

and

$$
\rho\left(v_{n}\right) \rightarrow \rho \quad w^{*} \text { in } L^{\infty}\left(0, T^{*} ; W^{1, q}\right), \text { as } n \rightarrow+\infty .
$$

Denoting by $u_{n}=\mathcal{T}\left(v_{n}, \rho\left(v_{n}\right)\right)$, by virtue of Lemmas 3.3-3.6 and Lemma 2.2 we get that, up to a subsequence,

$$
u_{n_{i}} \rightarrow u \quad w^{*} \text { in } L^{\infty}\left(0, T^{*} ; H_{0}^{1} \cap D^{2}\right) \cap L^{2}\left(0, T^{*} ; D^{2, q}\right), \text { as } n_{i} \rightarrow+\infty,
$$


and

$$
u_{n_{i}} \rightarrow u \quad \text { in } \mathcal{B} \text {, as } n_{i} \rightarrow+\infty \text {. }
$$

Letting $n_{i} \rightarrow+\infty$ in (3.7) where $\rho, u, v$ are replaced by $\rho\left(v_{n_{i}}\right), u_{n_{i}}, v_{n_{i}}$ respectively, we obtain that $u$ is a weak solution to (3.7) by (4.11), (4.8), (4.4), and (4.6), which again yields the uniqueness of the weak solutions to (3.7), and then $u=\mathcal{T}(v, \rho(v))$, which implies (4.11) holds for $u_{n}$ itself. That means $\mathcal{T}$ is a continuous operator in $\mathcal{B}$.

By Schauder fixed point theorem, Lemma 2.3, there exists $u \in \Re$ such that $\mathcal{T}(u, \rho(u))=u$, which together with Lemmas 3.3-3.5 implies (4.3). The uniqueness of $u$ and (4.2) follow from (4.3), standard Sobolev embedding theory, and Lemma 2.1.

Proposition 4.2. For $\tilde{\rho}=0$ and $\Omega=\mathbb{R}^{2}$, assume that $\left(\rho_{0} \geq 0, u_{0}\right)$ satisfies the conditions of Theorem 1.1. Then for $T^{*}$ as in Lemma 4.1, there exists a unique strong solution $(\rho, u)$ to the problem (1.1), (1.3), (1.4) on $\mathbb{R}^{2} \times\left[0, T^{*}\right]$, satisfying (4.2) and (4.3) with $\Omega=\mathbb{R}^{2}, q=4 / \alpha$ and any $p \in[2, q]$.

Proof. Define $B_{R}=\{x|| x \mid<R\}$ and

$$
\psi^{R}(x)=\psi(x / R), g^{R}(x)=\psi^{R}(x) g(x), \rho_{0}^{R}=\rho_{0}+R^{-2},
$$

for $(t, x) \in \mathbb{R}^{2} \times\left[0, T^{*}\right]$, where $0 \leq \psi \in C_{0}^{\infty}\left(B_{1}\right)$ is a smooth cut-off function such that $\psi=1$ in $B_{1 / 2}$. Let $u_{0}^{R} \in H_{0}^{1}\left(B_{R}\right) \cap H^{3}\left(B_{R}\right)$ be the unique solution to the elliptic boundary value problem

$$
L u_{0}^{R}=F_{0}^{R} \quad \text { in } \quad B_{R} \quad \text { and }\left.\quad u_{0}^{R}\right|_{\partial B_{R}}=0,
$$

where

$$
F_{0}^{R}=-\nabla P\left(\rho_{0}^{R}\right)+\sqrt{\rho_{0}^{R}} g^{R} .
$$

Extending $u_{0}^{R}$ to $\mathbb{R}^{2}$ by defining it to be zero outside $B_{R}$, we can show that

$$
u_{0}^{R} \rightarrow u_{0} \quad \text { in } \quad D^{1}\left(B_{R}\right) \quad \text { as } \quad R \rightarrow \infty .
$$

The proof is similar as in [4], so we omit it here.

By Lemma 4.1, there exists some $T^{*}>0$ such that for each $R>1$, the initialboundary-value problem (1.1), (4.1), with $\Omega=B_{R}$ and $\left(\rho_{0}, u_{0}\right)=\left(\rho_{0}^{R}, u_{0}^{R}\right)$ for $\rho_{0}^{R}, u_{0}^{R}$ defined as above, has a unique strong solution $(\rho, u)$ satisfying (4.2) and (4.3). We denote such $(\rho, u)$ by $\left(\rho^{R}, u^{R}\right)$.

Extending $\left(\rho^{R}, u^{R}\right)$ to $\mathbb{R}^{2}$ by zero outside $B_{R}$, we have from (4.3) that for $p \in[2, q]$,

$$
\begin{aligned}
& \sup _{0 \leq t \leq T^{*}}\left\|\psi^{R} \rho^{R}\right\|_{W^{1, p}}^{2} \\
\leq & C \sup _{0 \leq t \leq T^{*}} \int_{B_{R}}\left(\rho^{R}\right)^{p} d x+C \sup _{0 \leq t \leq T^{*}} \int_{B_{R}}\left|\nabla \rho^{R}\right|^{p} d x \\
\leq & C,
\end{aligned}
$$

and

$$
\sup _{0 \leq t \leq T^{*}} \int_{\mathbb{R}^{2}}\left|\left(\psi^{R} \rho^{R}\right)_{t}\right|^{p} d x \leq C \sup _{0 \leq t \leq T^{*}} \int_{B_{R}}\left|\rho_{t}^{R}\right|^{p} d x \leq C .
$$


Moreover, (4.3) gives

$$
\begin{aligned}
& \sup _{0 \leq t \leq T^{*}}\left\|\nabla\left(\psi^{R} u^{R}\right)\right\|_{H^{1}\left(\mathbb{R}^{2}\right)}^{2}+\sup _{0 \leq t \leq T^{*}}\left\|\psi^{R} u^{R}\right\|_{L^{q}\left(\mathbb{R}^{2}\right)}^{2} \\
\leq & C \sup _{0 \leq t \leq T^{*}} \int_{\mathbb{R}^{2}}\left|\nabla^{2} \psi^{R}\right|^{2}\left|u^{R}\right|^{2} d x+C \sup _{0 \leq t \leq T^{*}} \int_{\mathbb{R}^{2}}\left|\nabla \psi^{R}\right|^{2}\left|\nabla u^{R}\right|^{2} d x \\
& +C \sup _{0 \leq t \leq T^{*}} \int_{\mathbb{R}^{2}} \psi^{R}\left|\nabla^{2} u^{R}\right|^{2} d x+C \sup _{0 \leq t \leq T^{*}} \int_{\mathbb{R}^{2}} \psi^{R}\left|\nabla u^{R}\right|^{2} d x \\
& +C \sup _{0 \leq t \leq T^{*}} \int_{\mathbb{R}^{2}}\left|\nabla \psi^{R}\right|^{2}\left|u^{R}\right|^{2} d x+C \sup _{0 \leq t \leq T^{*}}\left\|u^{R}\right\|_{L^{q}\left(B_{R}\right)}^{2} \\
\leq & C R^{-2} \sup _{0 \leq t \leq T^{*}} \int_{B_{R}}\left|u^{R}\right|^{2} d x+C \sup _{0 \leq t \leq T^{*}} \int_{B_{R}}\left|\nabla u^{R}\right|^{2} d x \\
& +C \sup _{0 \leq t \leq T^{*}} \int_{B_{R}}\left|\nabla^{2} u^{R}\right|^{2} d x+C \sup _{0 \leq t \leq T^{*}}\left\|u^{R}\right\|_{L^{q}\left(B_{R}\right)}^{2} \\
\leq & C R^{-2+2(q-2) / q} \sup _{0 \leq t \leq T^{*}}\left\|u^{R}\right\|_{L^{q}\left(B_{R}\right)}^{2}+C \sup _{0 \leq t \leq T^{*}}\left\|u^{R}\right\|_{L^{q}\left(B_{R}\right)}^{2}+C \\
\leq & C \sup _{0 \leq t \leq T^{*}} \int_{B_{R}}|x|^{\alpha}\left|\nabla u^{R}\right|^{2} d x+C \\
\leq & C,
\end{aligned}
$$

and

$$
\begin{aligned}
& \int_{0}^{T^{*}}\left\|\left(\psi^{R} u^{R}\right)_{t}\right\|_{L^{q}\left(\mathbb{R}^{2}\right)}^{2} d t \\
\leq & C \int_{0}^{T^{*}}\left\|u_{t}^{R}\right\|_{L^{q}\left(B_{R}\right)}^{2} d t \\
\leq & C \int_{0}^{T^{*}}\left\|u^{R}\right\|_{L^{q}\left(B_{R}\right)}^{2} d t+C \int_{0}^{T^{*}}\left\|u^{R} \cdot \nabla u^{R}\right\|_{L^{q}\left(B_{R}\right)}^{2} d t \\
\leq & C+C \int_{0}^{T^{*}}\left\|u^{R}\right\|_{L^{\infty}\left(B_{R}\right)}^{2}\left\|\nabla u^{R}\right\|_{L^{q}\left(B_{R}\right)}^{2} d t \\
\leq & C .
\end{aligned}
$$

Next, (4.17) and (4.3) yield

$$
\begin{aligned}
& \int_{0}^{T^{*}}\left\|\nabla\left(\psi^{R} u^{R}\right)_{t}\right\|_{L^{2}\left(\mathbb{R}^{2}\right)}^{2} d t \\
\leq & \int_{0}^{T^{*}}\left\|\nabla \psi^{R}\right\| u_{t}^{R}\left\|_{L^{2}\left(B_{R}\right)}^{2} d t+\int_{0}^{T^{*}}\right\| \psi^{R} \nabla u_{t}^{R} \|_{L^{2}\left(B_{R}\right)}^{2} d t \\
\leq & C R^{-2} \int_{0}^{T^{*}}\left\|u_{t}^{R}\right\|_{L^{2}\left(B_{R}\right)}^{2} d t+\int_{0}^{T^{*}}\left\|\nabla u^{R}\right\|_{L^{2}\left(B_{R}\right)}^{2}+\left\|\nabla\left(u^{R} \cdot \nabla u^{R}\right)\right\|_{L^{2}\left(B_{R}\right)}^{2} d t \\
\leq & C R^{-4 / q} \int_{0}^{T^{*}}\left\|u_{t}^{R}\right\|_{L^{q}\left(B_{R}\right)}^{2} d t+C \int_{0}^{T^{*}}\left\|\nabla u^{R}\right\|_{L^{4}\left(B_{R}\right)}^{4} d t \\
& +C \int_{0}^{T^{*}}\left\|u^{R}\right\|_{L^{\infty}}^{2}\left\|\nabla^{2} u^{R}\right\|_{L^{2}\left(B_{R}\right)}^{2} d t+C \\
\leq & C+C \int_{0}^{T^{*}}\left\|\nabla u^{R}\right\|_{L^{2}\left(B_{R}\right)}^{2}\left\|\nabla^{2} u^{R}\right\|_{L^{2}\left(B_{R}\right)}^{2} d t
\end{aligned}
$$




$$
\leq C \text {. }
$$

Therefore, by Lemma 2.2 and a diagonalization procedure, we can choose a subsequence of $\left(\rho^{R}, u^{R}\right)$, still denoted by $\left(\rho^{R}, u^{R}\right)$, such that as $R \rightarrow \infty$

$$
\begin{aligned}
& \psi^{R} \rho^{R} \rightarrow \rho \quad w^{*} \text { in } L^{\infty}\left(0, T^{*} ; W^{1, q}\left(\mathbb{R}^{2}\right)\right) \cap W^{1, \infty}\left(0, T^{*} ; L^{p}\left(\mathbb{R}^{2}\right)\right), \\
& \psi^{R} \rho^{R} \rightarrow \rho \text { in } C\left(\overline{B_{n}} \times\left[0, T^{*}\right]\right), \\
& \psi^{R} u^{R} \rightarrow u \quad w^{*} \text { in } L^{\infty}\left(0, T^{*} ; D^{1}\left(\mathbb{R}^{2}\right) \cap D^{2}\left(\mathbb{R}^{2}\right)\right), \\
& \psi^{R} u^{R} \rightarrow u \quad w \text { in } H^{1}\left(0, T^{*} ; L^{q}\left(\mathbb{R}^{2}\right) \cap D^{1}\left(\mathbb{R}^{2}\right)\right),
\end{aligned}
$$

and

$$
\psi^{R} u^{R} \rightarrow u \text { in } C\left(\left[0, T^{*}\right] ; W^{1, q}\left(B_{n}\right)\right),
$$

for any positive integer $n$.

Now, for any function $\phi \in C_{0}^{\infty}\left(\mathbb{R}^{2} \times\left[0, T^{*}\right)\right)$, we take $\left(\psi^{R}\right)^{r} \phi$ with $r \geq \max \{\gamma+1,4\}$ as test function in (1.1), (4.1). Then letting $R \rightarrow \infty$, it follows from (4.12)-(4.23) that $(\rho, u)$ is a unique strong solution of (1.1), (1.3), (1.4) on $\mathbb{R}^{2} \times\left[0, T^{*}\right]$ satisfying (4.2), (4.3) with $\Omega=\mathbb{R}^{2}$.

The following Lemmas 4.3-4.6 deal with the higher order estimates of the unique strong solution $(\rho, u)$, obtained by Proposition 4.2 for $\tilde{\rho}=0$.

LEMma 4.3. The following estimates hold

$$
\begin{gathered}
\sup _{0 \leq t \leq T^{*}} \int \rho\left|u_{t}\right|^{2} d x+\int_{0}^{T^{*}}\left(\left\|u_{t}\right\|_{L^{q}}^{2}+\left\|\nabla u_{t}\right\|_{L^{2}}^{2}\right) d t \leq C, \\
\sup _{0 \leq t \leq T^{*}}\|(\rho, P)\|_{H^{2}}+\int_{0}^{T^{*}}\|\nabla u\|_{H^{2}}^{2} d t \leq C .
\end{gathered}
$$

Proof. It follows from (4.3) and (2.1) that

$$
\sup _{0 \leq t \leq T^{*}}\|u\|_{L^{q} \cap L^{\infty}} \leq C .
$$

Estimate (4.24) follows directly from the following simple facts:

$$
\begin{aligned}
\int \rho\left|u_{t}\right|^{2} d x & \leq \int \rho|\dot{u}|^{2} d x+\int \rho|u \cdot \nabla u|^{2} d x \\
& \leq C+C\|\rho\|_{L^{\infty}}\|u\|_{L^{\infty}}^{2}\|\nabla u\|_{L^{2}}^{2} \\
& \leq C,
\end{aligned}
$$




$$
\begin{aligned}
& \int_{0}^{T^{*}}\left\|u_{t}\right\|_{L^{q}}^{2} d t \\
\leq & C \int_{0}^{T^{*}}\|\dot{u}\|_{L^{q}}^{2} d t+C \int_{0}^{T^{*}}\|u \cdot \nabla u\|_{L^{q}}^{2} d t \\
\leq & C \int_{0}^{T^{*}}\left\||x|^{\alpha / 2} \nabla \dot{u}\right\|_{L^{2}}^{2} d t+C \int_{0}^{T^{*}}\|u\|_{L^{\infty}}^{2}\|\nabla u\|_{H^{1}}^{2} d t \\
\leq & C
\end{aligned}
$$

and

$$
\begin{aligned}
\left\|\nabla u_{t}\right\|_{L^{2}}^{2} & \leq\|\nabla \dot{u}\|_{L^{2}}^{2}+\|\nabla(u \cdot \nabla u)\|_{L^{2}}^{2} \\
& \leq\|\nabla \dot{u}\|_{L^{2}}^{2}+C\|u\|_{L^{\infty}}^{2}\left\|\nabla^{2} u\right\|_{L^{2}}^{2}+C\|\nabla u\|_{L^{4}}^{4} \\
& \leq\|\nabla \dot{u}\|_{L^{2}}^{2}+C
\end{aligned}
$$

due to (4.3), (4.26), and (2.2).

Next, we prove (4.25). Note that $P$ satisfies

$$
P_{t}+u \cdot \nabla P+\gamma P \operatorname{div} u=0
$$

which, together with $(1.1)_{1}$ and a simple computation, yields that

$$
\begin{aligned}
& \frac{d}{d t}\left(\left\|\nabla^{2} P\right\|_{L^{2}}+\left\|\nabla^{2} \rho\right\|_{L^{2}}\right) \\
\leq & C\left(1+\|\nabla u\|_{L^{\infty}}\right)\left(\left\|\nabla^{2} P\right\|_{L^{2}}+\left\|\nabla^{2} \rho\right\|_{L^{2}}\right)+C\left\|\nabla^{2} u\right\|_{H^{1}}+C\|\| \nabla^{2} u|(|\nabla \rho|+|\nabla P|)| \|_{L^{2}} \\
\leq & C\left(1+\|\nabla u\|_{L^{\infty}}\right)\left(\left\|\nabla^{2} P\right\|_{L^{2}}+\left\|\nabla^{2} \rho\right\|_{L^{2}}\right)+C\left\|\nabla^{2} u\right\|_{H^{1}}\left(1+\|\nabla \rho\|_{L^{q}}+\|\nabla P\|_{L^{q}}\right) \\
\leq & C\left(1+\|\nabla u\|_{W^{1, q}}\right)\left(\left\|\nabla^{2} P\right\|_{L^{2}}+\left\|\nabla^{2} \rho\right\|_{L^{2}}\right)+C\left\|\nabla^{2} u\right\|_{H^{1}} \\
\leq & C\left(1+\|\nabla u\|_{W^{1, q}}\right)\left(\left\|\nabla^{2} P\right\|_{L^{2}}+\left\|\nabla^{2} \rho\right\|_{L^{2}}\right)+C\left(1+\left\|\left.x\right|^{\alpha / 2} \nabla \dot{u}\right\|_{L^{2}}+\|\nabla \dot{u}\|_{L^{2}}\right),(4.28)
\end{aligned}
$$

where we have used the following standard $H^{1}$-estimate for elliptic system (3.45):

$$
\begin{aligned}
\left\|\nabla^{2} u\right\|_{H^{1}} & \leq C\left(\|\rho \dot{u}\|_{H^{1}}+\|\nabla P\|_{H^{1}}\right) \\
& \leq C\left(1+\left\|\rho^{1 / 2} \dot{u}\right\|_{L^{2}}+\|\nabla \rho\| \dot{u}\|\|_{L^{2}}+\|\rho \nabla \dot{u}\|_{L^{2}}+\left\|\nabla^{2} P\right\|_{L^{2}}\right) \\
& \leq C\left(1+\|\nabla \rho\|_{L^{2 q /(q-2)}}\|\dot{u}\|_{L^{q}}+\|\nabla \dot{u}\|_{L^{2}}+\left\|\nabla^{2} P\right\|_{L^{2}}\right) \\
& \leq C\left(1+\left\|\left.x\right|^{\alpha / 2} \nabla \dot{u}\right\|_{L^{2}}+\|\nabla \dot{u}\|_{L^{2}}+\left\|\nabla^{2} P\right\|_{L^{2}}\right),
\end{aligned}
$$

due to $(4.3)$ and $2 q /(q-2) \in(2, q)$. It thus follows from (4.3), (4.28), and Gronwall's inequality that

$$
\sup _{t \in\left[0, T^{*}\right]}\left(\left\|\nabla^{2} P\right\|_{L^{2}}+\left\|\nabla^{2} \rho\right\|_{L^{2}}\right) \leq C,
$$

which together with (4.3) and (4.29) implies (4.25). 
Lemma 4.4. The following estimates hold:

$$
\begin{gathered}
\sup _{0 \leq t \leq T^{*}}\left(\left\|\rho_{t}\right\|_{H^{1}}+\left\|P_{t}\right\|_{H^{1}}\right)+\int_{0}^{T^{*}}\left(\left\|\rho_{t t}\right\|_{L^{2}}^{2}+\left\|P_{t t}\right\|_{L^{2}}^{2}\right) d t \leq C, \\
\sup _{0 \leq t \leq T^{*}}\left\|\nabla u_{t}\right\|_{L^{2}}+\int_{0}^{T^{*}} \int \rho u_{t t}^{2} d x d t \leq C .
\end{gathered}
$$

Proof. We first prove (4.30). One deduces from (4.27), (4.3), and (4.26) that

$$
\left\|P_{t}\right\|_{L^{2}} \leq C\|u\|_{L^{\infty}}\|\nabla P\|_{L^{2}}+C\|\nabla u\|_{L^{2}} \leq C .
$$

Differentiating (4.27) yields

$$
\nabla P_{t}+u \cdot \nabla \nabla P+\nabla u \cdot \nabla P+\gamma \nabla P \operatorname{div} u+\gamma P \nabla \operatorname{div} u=0 .
$$

Hence, by (4.3), (4.26), and (4.25), one gets

$$
\left\|\nabla P_{t}\right\|_{L^{2}} \leq C\|u\|_{L^{\infty}}\left\|\nabla^{2} P\right\|_{L^{2}}+C\|\nabla u\|_{L^{4}}\|\nabla P\|_{L^{4}}+C\left\|\nabla^{2} u\right\|_{L^{2}} \leq C .
$$

The combination of (4.32) with (4.33) implies

$$
\sup _{0 \leq t \leq T^{*}}\left\|P_{t}\right\|_{H^{1}} \leq C .
$$

Note that $P_{t t}$ satisfies

$$
P_{t t}+\gamma P_{t} \operatorname{div} u+\gamma P \operatorname{div} u_{t}+u_{t} \cdot \nabla P+u \cdot \nabla P_{t}=0 .
$$

Thus, one gets from (4.35), (4.34), (4.3), and (4.24)-(4.26) that

$$
\begin{aligned}
& \int_{0}^{T^{*}}\left\|P_{t t}\right\|_{L^{2}}^{2} d t \\
\leq & C \int_{0}^{T^{*}}\left(\left\|P_{t}\right\|_{L^{4}}\|\nabla u\|_{L^{4}}+\left\|\nabla u_{t}\right\|_{L^{2}}+\left\|u_{t}\right\|_{L^{q}}\|\nabla P\|_{L^{2 q /(q-2)}}+\left\|\nabla P_{t}\right\|_{L^{2}}\right)^{2} d t \\
\leq & C .
\end{aligned}
$$

One can handle $\rho_{t}$ and $\rho_{t t}$ similarly. Hence (4.30) is proved.

Next, we prove (4.31). Differentiating $(1.1)_{2}$ with respect to $t$, then multiplying the resulting equation by $u_{t t}$, one gets after integration by parts that

$$
\begin{aligned}
& \frac{d}{d t} \int\left(\frac{\mu}{2}\left|\nabla u_{t}\right|^{2}+\frac{\lambda+\mu}{2}\left(\operatorname{div} u_{t}\right)^{2}\right) d x+\int \rho u_{t t}^{2} d x \\
= & \frac{d}{d t}\left(-\frac{1}{2} \int \rho_{t}\left|u_{t}\right|^{2} d x-\int \rho_{t} u \cdot \nabla u \cdot u_{t} d x+\int P_{t} \operatorname{div} u_{t} d x\right) \\
& +\frac{1}{2} \int \rho_{t t}\left|u_{t}\right|^{2} d x+\int\left(\rho_{t} u \cdot \nabla u\right)_{t} \cdot u_{t} d x-\int \rho u_{t} \cdot \nabla u \cdot u_{t t} d x \\
& -\int \rho u \cdot \nabla u_{t} \cdot u_{t t} d x-\int P_{t t} \operatorname{div} u_{t} d x
\end{aligned}
$$




$$
\triangleq \frac{d}{d t} I_{0}+\sum_{i=1}^{5} I_{i} .
$$

It follows from $(1.1)_{1},(4.3),(4.30),(4.24)$, and (4.26) that

$$
\begin{aligned}
\left|I_{0}\right|= & \left.\left|-\frac{1}{2} \int \rho_{t}\right| u_{t}\right|^{2} d x-\int \rho_{t} u \cdot \nabla u \cdot u_{t} d x+\int P_{t} \operatorname{div} u_{t} d x \mid \\
\leq & \left.\left|\int \operatorname{div}(\rho u)\right| u_{t}\right|^{2} d x|+C| \int \rho u \cdot \nabla\left(u \cdot \nabla u \cdot u_{t}\right) d x \mid+C\left\|P_{t}\right\|_{L^{2}}\left\|\nabla u_{t}\right\|_{L^{2}} \\
\leq & C \int \rho\left|u\left\|\left.u_{t}\left|\left(\left|\nabla u_{t}\right|+|\nabla u|^{2}+\left|u \| \nabla^{2} u\right|\right) d x+C \int \rho\right| u\right|^{2}|\nabla u|\left|\nabla u_{t}\right| d x+C\right\| \nabla u_{t} \|_{L^{2}}\right. \\
\leq & C\left\|\rho^{1 / 2} u_{t}\right\|_{L^{2}}\|u\|_{L^{\infty}}\left(\left\|\nabla u_{t}\right\|_{L^{2}}+\|\nabla u\|_{L^{4}}^{2}+\|u\|_{L^{\infty}}\left\|\nabla^{2} u\right\|_{L^{2}}\right) \\
& +C\|u\|_{L^{\infty}}^{2}\|\nabla u\|_{L^{2}}\left\|\nabla u_{t}\right\|_{L^{2}}+C\left\|\nabla u_{t}\right\|_{L^{2}} \\
\leq & \delta\left\|\nabla u_{t}\right\|_{L^{2}}^{2}+C_{\delta},
\end{aligned}
$$

and

$$
\begin{aligned}
& 2\left|I_{1}\right|=\left.\left|\int \rho_{t t}\right| u_{t}\right|^{2} d x \mid \\
&=\left|\int\left(\rho_{t} u+\rho u_{t}\right) \cdot \nabla\left(\left|u_{t}\right|^{2}\right) d x\right| \\
& \leq C\left\|\rho_{t}\right\|_{L^{2 q /(q-2)}}\|u\|_{L^{\infty}}\left\|u_{t}\right\|_{L^{q}}\left\|\nabla u_{t}\right\|_{L^{2}} \\
& \quad+C\left\|\rho^{1 / 2} u_{t}\right\|_{L^{2}}^{(q-4) /(q-2)}\left\|u_{t}\right\|_{L^{q}}^{q /(q-2)}\left\|\nabla u_{t}\right\|_{L^{2}} \\
& \leq C\left\|u_{t}\right\|_{L^{q}}^{2}+C\left\|\nabla u_{t}\right\|_{L^{2}}^{2}+C\left\|\nabla u_{t}\right\|_{L^{2}}^{2(q-2) /(q-4)}+C \\
& \leq C\left\|u_{t}\right\|_{L^{q}}^{2}+C\left\|\nabla u_{t}\right\|_{L^{2}}^{4}+C,
\end{aligned}
$$

due to $2(q-2) /(q-4) \in(2,4)$ by $(1.8)$. Moreover,

$$
\begin{aligned}
\left|I_{2}\right| & =\left|\int\left(\rho_{t} u \cdot \nabla u\right)_{t} \cdot u_{t} d x\right| \\
= & \left|\int\left(\rho_{t t} u \cdot \nabla u \cdot u_{t}+\rho_{t} u_{t} \cdot \nabla u \cdot u_{t}+\rho_{t} u \cdot \nabla u_{t} \cdot u_{t}\right) d x\right| \\
\leq & C\left\|\rho_{t t}\right\|_{L^{2}}\|u \cdot \nabla u\|_{L^{2 q /(q-2)}}\left\|u_{t}\right\|_{L^{q}}+C\left\|\rho_{t}\right\|_{L^{2}}\left\|\left.u_{t}\right|^{2}\right\|_{L^{q / 2}}\|\nabla u\|_{L^{2 q /(q-4)}} \\
\quad & +C\left\|\rho_{t}\right\|_{L^{2 q /(q-2)}}\|u\|_{L^{\infty}}\left\|\nabla u_{t}\right\|_{L^{2}}\left\|u_{t}\right\|_{L^{q}} \\
\leq & =C \rho_{t t}\left\|_{L^{2}}^{2}+C\right\| u_{t}\left\|_{L^{q}}^{2}+C\right\| \nabla u_{t} \|_{L^{2}}^{2} .
\end{aligned}
$$

Cauchy's inequality gives

$$
\begin{aligned}
\left|I_{3}\right|+\left|I_{4}\right| & =\left|\int \rho u_{t} \cdot \nabla u \cdot u_{t t} d x\right|+\left|\int \rho u \cdot \nabla u_{t} \cdot u_{t t} d x\right| \\
& \leq C\left\|\rho^{1 / 2} u_{t t}\right\|_{L^{2}}\left(\left\|u_{t}\right\|\left\|_{L^{q}}\right\| \nabla u\left\|_{L^{2 q /(q-2)}}+\right\| u\left\|_{L^{\infty}}\right\| \nabla u_{t} \|_{L^{2}}\right) \\
& \leq \delta\left\|\rho^{1 / 2} u_{t t}\right\|_{L^{2}}^{2}+C_{\delta}\left\|u_{t}\right\|_{L^{q}}^{2}+C_{\delta}\left\|\nabla u_{t}\right\|_{L^{2}}^{2},
\end{aligned}
$$

and

$$
\left|I_{5}\right|=\left|\int P_{t t} \operatorname{div} u_{t} d x\right| \leq\left\|P_{t t}\right\|_{L^{2}}\left\|\operatorname{div} u_{t}\right\|_{L^{2}} \leq C\left\|P_{t t}\right\|_{L^{2}}^{2}+\left\|\nabla u_{t}\right\|_{L^{2}}^{2} .
$$


Taking into account the compatibility condition (1.6), we can define

$$
\dot{u}(x, t=0)=g,
$$

which implies

$$
\begin{aligned}
\left\|\nabla u_{t}(\cdot, 0)\right\|_{L^{2}} & =\left\|\nabla \dot{u}(\cdot, 0)-\nabla\left(u_{0} \cdot \nabla u_{0}\right)\right\|_{L^{2}} \\
& \leq\|\nabla g\|_{L^{2}}+C\left(\left\|\nabla u_{0}\right\|_{L^{2}}+\left\|u_{0}\right\|_{L^{\infty}}\right)\left\|\nabla^{2} u_{0}\right\|_{L^{2}} \\
& \leq\|\nabla g\|_{L^{2}}+C .
\end{aligned}
$$

Now, combining all the estimates (4.37)-(4.41), we immediately deduce from (4.36), (4.30), (4.24), and Gronwall's inequality that (4.31) holds.

\section{LEMma 4.5. It holds that}

$$
\sup _{t \in\left[0, T^{*}\right]}\left(\|\rho\|_{H^{3}}+\|P\|_{H^{3}}+\|\nabla u\|_{H^{2}}\right)+\int_{0}^{T^{*}}\left(\|\nabla u\|_{H^{3}}^{2}+\left\|\nabla u_{t}\right\|_{H^{1}}^{2}\right) d t \leq C .
$$

Proof. The standard $L^{2}$-estimate for elliptic system, (4.3), and Lemma 4.4 yield that for $p \in[q,+\infty)$,

$$
\begin{aligned}
\left\|\nabla^{2} u_{t}\right\|_{L^{2}} \leq & C\left\|\mu \Delta u_{t}+(\mu+\lambda) \nabla \operatorname{div} u_{t}\right\|_{L^{2}} \\
= & \left\|\rho u_{t t}+\rho_{t} u_{t}+\rho_{t} u \cdot \nabla u+\rho u_{t} \cdot \nabla u+\rho u \cdot \nabla u_{t}+\nabla P_{t}\right\|_{L^{2}} \\
\leq & C\left(\left\|\rho u_{t t}\right\|_{L^{2}}+\left\|\rho_{t}\right\|_{L^{2 p /(p-2)}}\left\|u_{t}\right\|_{L^{p}}+\left\|\rho_{t}\right\|_{L^{4}}\|u\|_{L^{\infty}}\|\nabla u\|_{L^{4}}\right) \\
& \quad+C\left(\left\|u_{t}\right\|_{L^{p}}\|\nabla u\|_{L^{2 p /(p-2)}}+\|u\|_{L^{\infty}}\left\|\nabla u_{t}\right\|_{L^{2}}+\left\|\nabla P_{t}\right\|_{L^{2}}\right) \\
\leq & C\left\|\rho^{1 / 2} u_{t t}\right\|_{L^{2}}+C\left\|u_{t}\right\|_{L^{p}}+C,
\end{aligned}
$$

We observe from (4.43), (4.31), and (4.24) that

$$
\int_{0}^{T^{*}}\left\|\nabla u_{t}\right\|_{H^{1}}^{2} d t \leq C
$$

The standard $L^{2}$-estimate for elliptic system yields that for $p \in[q,+\infty)$,

$$
\begin{aligned}
\left\|\nabla^{4} u\right\|_{L^{2}} & \leq C\left\|\nabla^{2}(\mu \Delta u+(\mu+\lambda) \nabla \operatorname{div} u)\right\|_{L^{2}} \\
& \leq C\left\|\nabla^{2}(\rho \dot{u})\right\|_{L^{2}}+C\left\|\nabla^{3} P\right\|_{L^{2}} \\
& \leq C+C\|\nabla u\|_{H^{2}}+C\left\|u_{t}\right\|_{L^{p}}+C\left\|\nabla u_{t}\right\|_{H^{1}}+C\left\|\nabla^{3} P\right\|_{L^{2}},
\end{aligned}
$$

where one has used the following simple facts:

$$
\begin{aligned}
& \left\|\nabla^{2}\left(\rho u_{t}\right)\right\|_{L^{2}} \\
\leq & C\left(\left\|\left|\nabla^{2} \rho\left\|u_{t}\left|\left\|_{L^{2}}+\right\|\right| \nabla \rho\right\| \nabla u_{t}\right|\right\|_{L^{2}}+\left\|\nabla^{2} u_{t}\right\|_{L^{2}}\right) \\
\leq & C\left(\left\|\nabla^{2} \rho\right\|_{L^{2}}\left(\left\|u_{t}\right\|_{L^{p}}+\left\|\nabla u_{t}\right\|_{H^{1}}\right)+\|\nabla \rho\|_{L^{4}}\left\|\nabla u_{t}\right\|_{L^{4}}+\left\|\nabla^{2} u_{t}\right\|_{L^{2}}\right) \\
\leq & C\left\|u_{t}\right\|_{L^{p}}+C\left\|\nabla u_{t}\right\|_{H^{1}}
\end{aligned}
$$

and

$$
\begin{aligned}
& \left\|\nabla^{2}(\rho u \cdot \nabla u)\right\|_{L^{2}} \\
\leq & C\left(\left\|\left|\nabla ^ { 2 } ( \rho u ) \left\|\nabla u\left|\left\|_{L^{2}}+\right\| \nabla(\rho u)\left\|\nabla^{2} u \mid\right\|_{L^{2}}+\left\|\nabla^{3} u\right\|_{L^{2}}\right)\right.\right.\right.\right. \\
\leq & C\left(\left\|\nabla^{2}(\rho u)\right\|_{L^{2}}\|\nabla u\|_{H^{2}}+\|\nabla(\rho u)\|_{L^{4}}\left\|\nabla^{2} u\right\|_{L^{4}}+\left\|\nabla^{3} u\right\|_{L^{2}}\right) \\
\leq & C\left(1+\left\|\nabla^{2} \rho\right\|_{L^{2}}\|u\|_{L^{\infty}}+\|\nabla \rho\|_{L^{4}}\|\nabla u\|_{L^{4}}+\left\|\nabla^{2} u\right\|_{L^{2}}\right)\|\nabla u\|_{H^{2}} \\
\leq & C\|\nabla u\|_{H^{2}}
\end{aligned}
$$


due to (4.25), (4.3), and (2.3). In view of (4.27), (4.25), and (4.45), we have

$$
\begin{aligned}
& \left(\left\|\nabla^{3} P\right\|_{L^{2}}^{2}\right)_{t} \\
\leq & C\left(\left\|\left|\nabla^{3} u\|\nabla P\|\left\|_{L^{2}}+\right\|\right| \nabla^{2} u\right\| \nabla^{2} P\left|\left\|_{L^{2}}+\right\|\right| \nabla u\left\|\nabla^{3} P\right\|_{L^{2}}+\left\|\nabla^{4} u\right\|_{L^{2}}\right)\left\|\nabla^{3} P\right\|_{L^{2}} \\
\leq & C\left(\left\|\nabla^{3} u\right\|_{L^{2}}\|\nabla P\|_{H^{2}}+\left\|\nabla^{2} u\right\|_{H^{2}}\left(1+\left\|\nabla^{2} P\right\|_{L^{2}}\right)\right. \\
\quad & \left.\quad\|\nabla u\|_{L^{\infty}}\left\|\nabla^{3} P\right\|_{L^{2}}+\left\|\nabla^{4} u\right\|_{L^{2}}\right)\left\|\nabla^{3} P\right\|_{L^{2}} \\
\leq & C\left(1+\|\nabla u\|_{H^{2}}\right)\left(1+\left\|\nabla^{3} P\right\|_{L^{2}}^{2}\right)+C\left(\left\|\nabla^{2} u\right\|_{H^{2}}+\left\|\nabla^{4} u\right\|_{L^{2}}\right)\left\|\nabla^{3} P\right\|_{L^{2}} \\
\leq & C\left(1+\|\nabla u\|_{H^{2}}+\left\|u_{t}\right\|_{L^{q}}+\left\|\nabla u_{t}\right\|_{H^{1}}\right)\left(1+\left\|\nabla^{3} P\right\|_{L^{2}}^{2}\right),
\end{aligned}
$$

which, together with Gronwall's inequality and (4.25), (4.24), (4.44), yields that

$$
\sup _{0 \leq t \leq T^{*}}\left\|\nabla^{3} P\right\|_{L^{2}} \leq C .
$$

It thus follows from (4.44)-(4.46), (4.24), (4.25), and (4.3) that

$$
\int_{0}^{T^{*}}\|\nabla u\|_{H^{3}}^{2} d t \leq C
$$

which, together with (4.46) and (4.25), shows that

$$
\sup _{0 \leq t \leq T^{*}}\|P\|_{H^{3}}+\int_{0}^{T^{*}}\|\nabla u\|_{H^{3}}^{2} d t \leq C .
$$

It is easy to check that similar arguments work for $\rho$ by using (4.47). Hence,

$$
\sup _{0 \leq t \leq T^{*}}\|\rho\|_{H^{3}} \leq C
$$

Furthermore, it follows directly from (4.44) and (4.47) that

$$
\sup _{0 \leq t \leq T^{*}}\left\|\nabla^{3} u\right\|_{L^{2}} \leq C \text {. }
$$

Thus (4.42) holds by (4.47), (4.48), (4.44), (4.49), and (4.3).

LEMma 4.6. The following estimate holds:

$$
\sup _{0 \leq t \leq T^{*}} t\left(\left\|u_{t}\right\|_{L^{8 / \alpha} \cap D^{2}}^{2}+\left\|\nabla^{4} u\right\|_{L^{2}}^{2}+\left\|\rho^{1 / 2} u_{t t}\right\|_{L^{2}}^{2}\right)+\int_{0}^{T^{*}} t\left\|\nabla u_{t t}\right\|_{L^{2}}^{2} d t \leq C .
$$

Proof. Differentiate $(1.1)_{2}$ with respect to $t$ twice to get

$$
\begin{aligned}
& \rho u_{t t t}+\rho u \cdot \nabla u_{t t}-\mu \Delta u_{t t}-(\mu+\lambda) \nabla \operatorname{div} u_{t t} \\
= & 2 \operatorname{div}(\rho u) u_{t t}+\operatorname{div}(\rho u)_{t} u_{t}-2(\rho u)_{t} \cdot \nabla u_{t}-\left(\rho_{t t} u+2 \rho_{t} u_{t}\right) \cdot \nabla u-\rho u_{t t} \cdot \nabla u-\nabla P_{t t} .
\end{aligned}
$$

Multiplying (4.51) by $u_{t t}$ and then integrating the resulting equation over $\mathbb{R}^{2}$, one gets after integration by parts that

$$
\frac{1}{2} \frac{d}{d t} \int \rho\left|u_{t t}\right|^{2} d x+\int\left(\mu\left|\nabla u_{t t}\right|^{2}+(\mu+\lambda)\left(\operatorname{div} u_{t t}\right)^{2}\right) d x
$$




$$
\begin{aligned}
= & -4 \int u_{t t}^{i} \rho u \cdot \nabla u_{t t}^{i} d x-\int(\rho u)_{t} \cdot\left[\nabla\left(u_{t} \cdot u_{t t}\right)+2 \nabla u_{t} \cdot u_{t t}\right] d x \\
& -\int\left(\rho_{t t} u+2 \rho_{t} u_{t}\right) \cdot \nabla u \cdot u_{t t} d x-\int \rho u_{t t} \cdot \nabla u \cdot u_{t t} d x+\int P_{t t} \operatorname{div} u_{t t} d x \\
= & -4 \int u_{t t}^{i} \rho u \cdot \nabla u_{t t}^{i} d x-\int \rho u_{t} \cdot\left[\nabla\left(u_{t} \cdot u_{t t}\right)+2 \nabla u_{t} \cdot u_{t t}\right] d x \\
& -\int \rho_{t} u^{i} u_{t}^{j} \partial_{i} u_{t t}^{j} d x+3 \int \operatorname{div}(\rho u) u \cdot \nabla u_{t}^{j} u_{t t}^{j} d x \\
& +\int\left(\operatorname{div}(\rho u)_{t} u+2 \operatorname{div}(\rho u) u_{t}\right) \cdot \nabla u \cdot u_{t t} d x \\
& -\int \rho u_{t t} \cdot \nabla u \cdot u_{t t} d x+\int P_{t t} \operatorname{div} u_{t t} d x \\
= & -4 \int u_{t t}^{i} \rho u \cdot \nabla u_{t t}^{i} d x-\int \rho u_{t} \cdot\left[\nabla\left(u_{t} \cdot u_{t t}\right)+2 \nabla u_{t} \cdot u_{t t}\right] d x \\
& -\int \rho_{t} u^{i} u_{t}^{j} \partial_{i} u_{t t}^{j} d x-3 \int \rho u^{i} \partial_{i}\left(u \cdot \nabla u_{t}^{j} u_{t t}^{j}\right) d x \\
& -\int \rho u_{t}^{i} \partial_{i}\left(u^{k} \partial_{k} u^{j} u_{t t}^{j}\right) d x-\int \rho_{t} u^{i} u^{k} \partial_{k} u^{j} \partial_{i} u_{t t}^{j} d x \\
& +\int \operatorname{div}(\rho u) u^{i} \partial_{i}\left(u^{k} \partial_{k} u^{j}\right) u_{t t}^{j} d x-2 \int \rho u^{i} \partial_{i}\left(u_{t}^{k} \partial_{k} u^{j} u_{t t}^{j}\right) d x \\
& -\int \rho u_{t t} \cdot \nabla u \cdot u_{t t} d x+\int P_{t t} \operatorname{div} u_{t t} d x \\
\triangleq & \sum_{i=1}^{10} J_{i} .
\end{aligned}
$$

We estimate each $J_{i}(i=1, \cdots, 10)$ as follows. Hölder's inequality gives

$$
\begin{aligned}
\left|J_{1}\right| & \leq C\left\|\rho^{1 / 2} u_{t t}\right\|_{L^{2}}\left\|\nabla u_{t t}\right\|_{L^{2}}\|u\|_{L^{\infty}} \\
& \leq \delta\left\|\nabla u_{t t}\right\|_{L^{2}}^{2}+C_{\delta}\left\|\rho^{1 / 2} u_{t t}\right\|_{L^{2}}^{2} .
\end{aligned}
$$

It follows from (4.24), (4.30), (4.31), and (4.3) that

$$
\begin{aligned}
\left|J_{2}\right| & \leq C \int\left(\rho\left|u_{t}\right|^{2}\left|\nabla u_{t t}\right|+\rho\left|u_{t t}\right|\left|u_{t}\right|\left|\nabla u_{t}\right|\right) d x \\
& \leq C\left\|\rho^{1 / 2} u_{t}\right\|_{L^{2}}\left\|u_{t}\right\|_{L^{\infty}}\left\|\nabla u_{t t}\right\|_{L^{2}}+C\left\|\rho^{1 / 2} u_{t t}\right\|_{L^{2}}\left\|u_{t}\right\|_{L^{\infty}}\left\|\nabla u_{t}\right\|_{L^{2}} \\
& \leq \delta\left\|\nabla u_{t t}\right\|_{L^{2}}^{2}+C_{\delta}\left(\left\|u_{t}\right\|_{L^{q}}^{2}+\left\|\nabla u_{t}\right\|_{H^{1}}^{2}\right)+C\left\|\rho^{1 / 2} u_{t t}\right\|_{L^{2}}^{2},
\end{aligned}
$$

and

$$
\begin{aligned}
\left|J_{3}\right|+\left|J_{6}\right| & \leq C \int\left|\rho_{t}\|u\| \nabla u_{t t}\right|\left(\left|u_{t}\right|+|u||\nabla u|\right) d x \\
& \leq C\left(\left\|\rho_{t}\right\|_{L^{2 q /(q-2)}}\|u\|_{L^{\infty}}\left\|u_{t}\right\|_{L^{q}}+\left\|\rho_{t}\right\|_{L^{4}}\|u\|_{L^{\infty}}^{2}\|\nabla u\|_{L^{4}}\right)\left\|\nabla u_{t t}\right\|_{L^{2}} \\
& \leq \delta\left\|\nabla u_{t t}\right\|_{L^{2}}^{2}+C_{\delta}\left\|u_{t}\right\|_{L^{q}}^{2}+C_{\delta} .
\end{aligned}
$$

Next, we observe

$$
\left|J_{4}\right| \leq C \int \rho|u|\left|u_{t t}\right|\left(|\nabla u|\left|\nabla u_{t}\right|+|u|\left|\nabla^{2} u_{t}\right|\right) d x+C \int \rho|u|^{2}\left|\nabla u_{t}\right|\left|\nabla u_{t t}\right| d x
$$




$$
\begin{aligned}
& \leq C\left\|\rho^{1 / 2} u_{t t}\right\|_{L^{2}}\left(\|u\|_{L^{\infty}}\|\nabla u\|_{L^{4}}\left\|\nabla u_{t}\right\|_{L^{4}}+\|u\|_{L^{\infty}}^{2}\left\|\nabla^{2} u_{t}\right\|_{L^{2}}\right) \\
& \quad+C\|u\|_{L^{\infty}}^{2}\left\|\nabla u_{t}\right\|_{L^{2}}\left\|\nabla u_{t t}\right\|_{L^{2}} \\
& \leq \delta\left\|\nabla u_{t t}\right\|_{L^{2}}^{2}+C_{\delta}\left\|\nabla u_{t}\right\|_{H^{1}}^{2}+C\left\|\rho^{1 / 2} u_{t t}\right\|_{L^{2}}^{2}
\end{aligned}
$$

and

$$
\begin{aligned}
\left|J_{5}\right| \leq & C \int \rho\left|u_{t t}\left\|u_{t}\left|\left(|\nabla u|^{2}+\left|u \| \nabla^{2} u\right|\right) d x+C \int \rho\right| u_{t}\right\| u\|\nabla u\| \nabla u_{t t}\right| d x \\
\leq & C\left\|\rho^{1 / 2} u_{t t}\right\|_{L^{2}}\left\|u_{t}\right\|_{L^{q}}\left(\|\nabla u\|_{L^{4 q /(q-2)}}^{2}+\left\|\nabla^{2} u\right\|_{L^{2 q /(q-2)}}\|u\|_{L^{\infty}}\right) \\
& +C\left\|u_{t}\right\|_{L^{q}}\|u\|_{L^{\infty}}\|\nabla u\|_{L^{2 q /(q-2)}}\left\|\nabla u_{t t}\right\|_{L^{2}} \\
\leq & \delta\left\|\nabla u_{t t}\right\|_{L^{2}}^{2}+C_{\delta}\left\|u_{t}\right\|_{L^{q}}^{2}+C\left\|\rho^{1 / 2} u_{t t}\right\|_{L^{2}}^{2} .
\end{aligned}
$$

Moreover,

$$
\begin{aligned}
&\left|J_{7}\right|=\left|\int \rho u^{l} \partial_{l}\left(u^{i} \partial_{i}\left(u^{k} \partial_{k} u^{j}\right) u_{t t}^{j}\right) d x\right| \\
& \leq C \int \rho\left|u_{t t}\right||u|\left(|\nabla u|^{3}+|u||\nabla u|\left|\nabla^{2} u\right|+|u|^{2}\left|\nabla^{3} u\right|\right) d x \\
&+C \int \rho|u|^{2}\left|\nabla u_{t t}\right|\left(|\nabla u|^{2}+|u|\left|\nabla^{2} u\right|\right) d x \\
& \leq C\left\|\rho^{1 / 2} u_{t t}\right\|_{L^{2}}\left(\|\nabla u\|_{L^{6}}^{3}+\|\nabla u\|_{L^{4}}\left\|\nabla^{2} u\right\|_{L^{4}}+\left\|\nabla^{3} u\right\|_{L^{2}}\right) \\
&+C\left\|\nabla u_{t t}\right\|_{L^{2}}\left(\|\nabla u\|_{L^{4}}^{2}+\left\|\nabla^{2} u\right\|_{L^{2}}\right) \\
& \leq C\left\|\rho^{1 / 2} u_{t t}\right\|_{L^{2}}^{2}+\delta\left\|\nabla u_{t t}\right\|_{L^{2}}^{2}+C_{\delta}, \\
&\left|J_{8}\right| \leq C \int \rho\left|u_{t t}\right||u|\left(\left|\nabla u_{t}\right||\nabla u|+\left|u_{t} \| \nabla^{2} u\right|\right) d x+C \int \rho\left|u \| \nabla u_{t t}\right|\left|u_{t}\right||\nabla u| d x \\
& \leq C\left\|\rho^{1 / 2} u_{t t}\right\|_{L^{2}}\left(\left\|\nabla u_{t}\right\|_{L^{4}}\|\nabla u\|_{L^{4}}+\left\|u_{t}\right\|_{L^{q}}\left\|\nabla^{2} u\right\|_{L^{2 q /(q-2)}}\right) \\
&+C\left\|\nabla u_{t t}\right\|_{L^{2}}\left\|u_{t}\right\|_{L^{q}}\|\nabla u\|_{L^{2 q /(q-2)}} \\
& \leq C\left\|\rho^{1 / 2} u_{t t}\right\|_{L^{2}}^{2}+C\left\|\nabla u_{t}\right\|_{H^{1}}^{2}+C_{\delta}\left\|u_{t}\right\|_{L^{q}}^{2}+\delta\left\|\nabla u_{t t}\right\|_{L^{2}}^{2},
\end{aligned}
$$

and

$$
\begin{aligned}
\left|J_{9}\right|+\left|J_{10}\right| & \leq C\left\|\rho^{1 / 2} u_{t t}\right\|_{L^{2}}^{2}\|\nabla u\|_{L^{\infty}}+C\left\|P_{t t}\right\|_{L^{2}}\left\|\nabla u_{t t}\right\|_{L^{2}} \\
& \leq \delta\left\|\nabla u_{t t}\right\|_{L^{2}}^{2}+C_{\delta}\left\|\rho^{1 / 2} u_{t t}\right\|_{L^{2}}^{2}+C_{\delta}\left\|P_{t t}\right\|_{L^{2}}^{2} .
\end{aligned}
$$

Multiplying (4.52) by $t$ and integrating the resulting equation with respect to $t$ over $\left(0, T^{*}\right)$, we obtain, by using (4.53)-(4.60), (4.30), (4.31), and Gronwall's inequality after choosing $\delta$ suitably small, that

$$
\sup _{0 \leq t \leq T^{*}} \int t \rho\left|u_{t t}\right|^{2} d x+\int_{0}^{T^{*}} t \int\left|\nabla u_{t t}\right|^{2} d x d t \leq C .
$$

Next, setting $v=u$ in (3.34) and multiplying the resulting equation by $|x|^{\alpha / 2}(\dot{u})_{t}$, we obtain after integrating by parts that

$$
\int|x|^{\alpha / 2} \rho\left|(\dot{u})_{t}\right|^{2} d x+\frac{1}{2} \frac{d}{d t}\left(\mu \int|x|^{\alpha / 2}|\nabla \dot{u}|^{2} d x+(\mu+\lambda) \int|x|^{\alpha / 2}|\operatorname{div} \dot{u}|^{2} d x\right)
$$




$$
\begin{aligned}
= & -\int \rho(u \cdot \nabla \dot{u})|x|^{\alpha / 2}(\dot{u})_{t} d x \\
& -\frac{\mu \alpha}{2} \int \partial_{i} \dot{u}^{k}\left(\dot{u}^{k}\right)_{t}|x|^{\alpha / 2-2} x^{i} d x-\frac{\alpha(\mu+\lambda)}{2} \int \operatorname{div} \dot{u}\left((\dot{u})_{t} \cdot x\right)|x|^{\alpha / 2-2} d x \\
& +\mu \int\left(\partial_{i} u^{k} \partial_{i} u\right) \partial_{k}\left(|x|^{\alpha / 2}(\dot{u})_{t}\right) d x-\mu \int\left(\partial_{k} u^{k} \partial_{i} u\right) \partial_{i}\left(|x|^{\alpha / 2}(\dot{u})_{t}\right) d x \\
& +\mu \int\left(\partial_{i} u^{k} \partial_{k} u\right) \partial_{i}\left(|x|^{\alpha / 2}(\dot{u})_{t}\right) d x-(\mu+\lambda)\left[\int(\operatorname{div} u)^{2} \operatorname{div}\left(|\mathrm{x}|^{\alpha / 2}(\dot{\mathrm{u}})_{\mathrm{t}}\right) \mathrm{dx}\right. \\
& \left.-\int\left(\partial_{j} u^{k} \partial_{k} u^{j}\right) \operatorname{div}\left(|\mathrm{x}|^{\alpha / 2}(\dot{\mathrm{u}})_{\mathrm{t}}\right) \mathrm{dx}-\int\left(\nabla \mathrm{u}^{\mathrm{k}} \operatorname{divu}\right) \cdot \partial_{\mathrm{k}}\left(|\mathrm{x}|^{\alpha / 2}(\dot{\mathrm{u}})_{\mathrm{t}}\right) \mathrm{dx}\right] \\
& -\int\left(P \nabla u^{k}\right) \cdot \partial_{k}\left(|x|^{\alpha / 2}(\dot{u})_{t}\right) d x-(\gamma-1) \int P \operatorname{div} u \operatorname{div}\left(|x|^{\alpha / 2}(\dot{u})_{t}\right) d x \triangleq \sum_{i=1}^{9} K_{i} .
\end{aligned}
$$

In view of (2.1), Lemma 4.4 and 4.5, we estimate each term on the righthand side of (4.62) as follows:

$$
\begin{aligned}
\left|K_{1}\right| & =\left.\left|\int \rho(u \cdot \nabla \dot{u})\right| x\right|^{\alpha / 2}(\dot{u})_{t} d x \mid \\
& \leq C\|u\|_{L^{\infty}}\left\|\rho^{1 / 2}(\dot{u})_{t}|x|^{\alpha / 4}\right\|_{L^{2}}\left\|\left|\nabla \dot{u}\left\|\left.x\right|^{\alpha / 4}\right\|_{L^{2}}\right.\right. \\
& \leq \frac{1}{2}\left\|\rho^{1 / 2}(\dot{u})_{t}|x|^{\alpha / 4}\right\|_{L^{2}}^{2}+C\left\|\left|\nabla \dot{u}\left\|\left.x\right|^{\alpha / 4}\right\|_{L^{2}}^{2}\right.\right. \\
& \leq \frac{1}{2}\left\|\rho^{1 / 2}(\dot{u})_{t}|x|^{\alpha / 4}\right\|_{L^{2}}^{2}+C\left\|\left|\nabla \dot{u}\left\|\left.x\right|^{\alpha / 2}\right\|_{L^{2}}^{2}+C\|\nabla \dot{u}\|_{L^{2}}^{2},\right.\right.
\end{aligned}
$$

$$
\begin{aligned}
K_{2}= & -\frac{\mu \alpha}{2} \int \partial_{i} \dot{u}^{k}\left(\dot{u}^{k}\right)_{t}|x|^{\alpha / 2-2} x^{i} d x \\
\leq & -\frac{\mu \alpha}{2} \frac{d}{d t} \int \partial_{i} \dot{u}^{k} \dot{u}^{k}|x|^{\alpha / 2-2} x^{i} d x+C \int\left|\nabla(\dot{u})_{t}\|\dot{u}\| x\right|^{(\alpha-2) / 2} d x \\
\leq & -\frac{\mu \alpha}{2} \frac{d}{d t} \int \partial_{i} \dot{u}^{k} \dot{u}^{k}|x|^{\alpha / 2-2} x^{i} d x+C\left\|\nabla\left(u_{t t}+u_{t} \cdot \nabla u+u \cdot \nabla u_{t}\right)\right\|_{L^{2}}\left\|\dot{u}|x|^{(\alpha-2) / 2}\right\|_{L^{2}} \\
\leq & -\frac{\mu \alpha}{2} \frac{d}{d t} \int \partial_{i} \dot{u}^{k} \dot{u}^{k}|x|^{\alpha / 2-2} x^{i} d x \\
& +C\left(\left\|\left|\nabla \dot{u}\left\|\left.x\right|^{\alpha / 2}\right\|_{L^{2}}^{2}+\left\|\nabla u_{t t}\right\|_{L^{2}}^{2}+\left\|u_{t}\right\|_{L^{4 / \alpha}}^{2}\left\|\nabla^{2} u\right\|_{L^{4 /(2-\alpha)}}^{2}\right.\right.\right. \\
& \left.+\|\nabla u\|_{H^{2}}\left\|\nabla u_{t}\right\|_{L^{2}}^{2}+\|u\|_{L^{\infty}}^{2}\left\|\nabla^{2} u_{t}\right\|_{L^{2}}^{2}\right) \\
\leq & -\frac{\mu \alpha}{2} \frac{d}{d t} \int \partial_{i} \dot{u}^{k} \dot{u}^{k}|x|^{\alpha / 2-2} x^{i} d x \\
& +C\left(\left\|\left|\nabla \dot{u}\left\|\left.x\right|^{\alpha / 2}\right\|_{L^{2}}^{2}+\left\|\nabla u_{t t}\right\|_{L^{2}}^{2}+\left\|u_{t}\right\|_{L^{4 / \alpha}}^{2}+\left\|\nabla^{2} u_{t}\right\|_{L^{2}}^{2}+1\right),\right.\right. \\
K_{4}= & \mu \int\left(\partial_{i} u^{k} \partial_{i} u\right) \partial_{k}\left(|x|^{\alpha / 2}(\dot{u})_{t}\right) d x \\
\leq & \mu \frac{d}{d t} \int\left(\partial_{i} u^{k} \partial_{i} u\right) \partial_{k}\left(|x|^{\alpha / 2} \dot{u}\right) d x+C \int\left|\nabla u_{t} \| \nabla u\right|\left(\left|\nabla \dot{u}\left\|\left.x\right|^{\alpha / 2}+|\dot{u} \| x|^{(\alpha-2) / 2}\right) d x\right.\right. \\
\leq & \mu \frac{d}{d t} \int\left(\partial_{i} u^{k} \partial_{i} u\right) \partial_{k}\left(|x|^{\alpha / 2} \dot{u}\right) d x
\end{aligned}
$$




$$
\begin{aligned}
& +C\left(\|\nabla u\|_{L^{\infty}}\left\|\nabla u_{t}\right\|_{L^{2}}\right)\left(\left\|\nabla \dot{u}|x|^{\alpha / 2}\right\|_{L^{2}}+\left\|\dot{u}|x|^{(\alpha-2) / 2}\right\|_{L^{2}}\right) \\
& \leq \mu \frac{d}{d t} \int\left(\partial_{i} u^{k} \partial_{i} u\right) \partial_{k}\left(|x|^{\alpha / 2} \dot{u}\right) d x+C\left\|\nabla \dot{u}|x|^{\alpha / 2}\right\|_{L^{2}}^{2}+C,
\end{aligned}
$$

and

$$
\begin{aligned}
K_{8}= & -\int\left(P \nabla u^{k}\right) \cdot \partial_{k}\left(|x|^{\alpha / 2}(\dot{u})_{t}\right) d x \\
\leq & -\frac{d}{d t} \int\left(P \nabla u^{k}\right) \cdot \partial_{k}\left(|x|^{\alpha / 2} \dot{u}\right) d x \\
& +C \int\left(\left|P_{t}\right||\nabla u|+P\left|\nabla u_{t}\right|\right)\left(\left|\nabla \dot{u}\left\|\left.x\right|^{\alpha / 2}+|\dot{u} \| x|^{(\alpha-2) / 2}\right) d x\right.\right. \\
\leq & -\frac{d}{d t} \int\left(P \nabla u^{k}\right) \cdot \partial_{k}\left(|x|^{\alpha / 2} \dot{u}\right) d x+C\left(\left\|P_{t}\right\|_{L^{2}}\|\nabla u\|_{L^{\infty}}+\|P\|_{L^{\infty}}\left\|\nabla u_{t}\right\|_{L^{2}}\right) \\
& \times\left(\left\|\nabla \dot{u}|x|^{\alpha / 2}\right\|_{L^{2}}+\left\|\dot{u}|x|^{(\alpha-2) / 2}\right\|_{L^{2}}\right) \\
\leq & -\frac{d}{d t} \int\left(P \nabla u^{k}\right) \cdot \partial_{k}\left(|x|^{\alpha / 2} \dot{u}\right) d x+C\left\|\nabla \dot{u}|x|^{\alpha / 2}\right\|_{L^{2}}^{2}+C .
\end{aligned}
$$

Similarly, we can estimate the other terms on the righthand side of (4.62). Therefore, we arrive at

$$
\begin{aligned}
& \quad \frac{1}{2} \int|x|^{\alpha / 2} \rho\left|\dot{u}_{t}\right|^{2} d x+\frac{1}{2} \frac{d}{d t}\left(\mu \int|x|^{\alpha / 2}|\nabla \dot{u}|^{2} d x+(\mu+\lambda) \int|x|^{\alpha / 2}|\operatorname{div} \dot{u}|^{2} d x\right) \\
& \leq \frac{d}{d t} \Psi(t)+C\left(\|\nabla \dot{u}\|_{L^{2}}^{2}+\left\|\left|\nabla \dot{u}\left\|\left.x\right|^{\alpha / 2}\right\|_{L^{2}}^{2}+\left\|\nabla u_{t t}\right\|_{L^{2}}^{2}+\left\|u_{t}\right\|_{L^{4 / \alpha}}^{2}\right)\right.\right. \\
& \quad+C\left\|\nabla^{2} u_{t}\right\|_{L^{2}}^{2}+C,
\end{aligned}
$$

where

$$
\begin{aligned}
\Psi(t) \triangleq & -\frac{\mu \alpha}{2} \int \partial_{i} \dot{u}^{k} \dot{u}^{k}|x|^{\alpha / 2-2} x^{i} d x-\frac{\alpha(\mu+\lambda)}{2} \int \operatorname{div} \dot{u}(\dot{u} \cdot x)|x|^{\alpha / 2-2} d x \\
& +\mu \int\left(\partial_{i} u^{k} \partial_{i} u\right) \partial_{k}\left(|x|^{\alpha / 2} \dot{u}\right) d x-\mu \int\left(\partial_{k} u^{k} \partial_{i} u\right) \partial_{i}\left(|x|^{\alpha / 2} \dot{u}\right) d x \\
& +\mu \int\left(\partial_{i} u^{k} \partial_{k} u\right) \partial_{i}\left(|x|^{\alpha / 2} \dot{u}\right) d x-(\mu+\lambda)\left[\int(\operatorname{div} u)^{2} \operatorname{div}\left(|\mathrm{x}|^{\alpha / 2} \dot{\mathrm{u}}\right) \mathrm{dx}\right. \\
& \left.-\int\left(\partial_{j} u^{k} \partial_{k} u^{j}\right) \operatorname{div}\left(|\mathrm{x}|^{\alpha / 2} \dot{\mathrm{u}}\right) \mathrm{dx}-\int\left(\nabla \mathrm{u}^{\mathrm{k}} \operatorname{divu}\right) \cdot \partial_{\mathrm{k}}\left(|\mathrm{x}|^{\alpha / 2} \dot{\mathrm{u}}\right) \mathrm{dx}\right] \\
& -\int\left(P \nabla u^{k}\right) \cdot \partial_{k}\left(|x|^{\alpha / 2} \dot{u}\right) d x-(\gamma-1) \int P \operatorname{div} u \operatorname{div}\left(|x|^{\alpha / 2} \dot{u}\right) d x .
\end{aligned}
$$

It is easy to show that

$$
\begin{aligned}
|\Psi(t)| \leq & \mu \alpha^{2}\left\||x|^{\alpha / 4} \nabla \dot{u}\right\|_{L^{2}}^{2}+(\mu+\lambda) \alpha^{2}\left\||x|^{\alpha / 4} \nabla \dot{u}\right\|_{L^{2}}^{2} \\
& +C \int\left(|\nabla u|^{2}+|\nabla u|\right)\left(|x|^{\alpha / 2}|\nabla \dot{u}|+|\dot{u} \| x|^{(\alpha-2) / 2}\right) d x \\
\leq & (2 \mu+\lambda) \alpha^{2}\left\||x|^{\alpha / 4} \nabla \dot{u}\right\|_{L^{2}}^{2}+C \int|\nabla u|\left(|x|^{\alpha / 2}|\nabla \dot{u}|+|\dot{u} \| x|^{(\alpha-2) / 2}\right) d x \\
\leq & (2 \mu+\lambda) \alpha^{2}\left\||x|^{\alpha / 4} \nabla \dot{u}\right\|_{L^{2}}^{2}+C\left\|\nabla u|x|^{\alpha / 4}\right\|_{L^{2}}\left\|\left.x\right|^{\alpha / 4} \mid \nabla \dot{u}\right\|_{L^{2}} \\
\leq & 2(2 \mu+\lambda) \alpha^{2}\left\||x|^{\alpha / 4} \nabla \dot{u}\right\|_{L^{2}}^{2}+C\left\|\nabla u|x|^{\alpha / 2}\right\|_{L^{2}}^{2}+C\|\nabla u\|_{L^{2}}^{2}
\end{aligned}
$$




$$
\leq \frac{\mu}{8}\left\||x|^{\alpha / 4} \nabla \dot{u}\right\|_{L^{2}}^{2}+C .
$$

Multiplying (4.67) by $t$ and integrating the resulting equation over $\left(0, T^{*}\right)$, we deduce from (4.68), (4.61), (4.3), and (4.24) that

$$
\begin{aligned}
& \sup _{0 \leq t \leq T^{*}} \int t|x|^{\alpha / 2}|\nabla \dot{u}|^{2} d x+\int_{0}^{T^{*}} t \int|x|^{\alpha / 2} \rho\left|\dot{u}_{t}\right|^{2} d x d t \\
& \leq C \int_{0}^{T^{*}}\left(\|\nabla \dot{u}\|_{L^{2}}^{2}+\left\|\left|\nabla \dot{u}\left\|\left.x\right|^{\alpha / 2}\right\|_{L^{2}}^{2}\right.\right.\right. \\
& \left.\quad+t\left\|\nabla u_{t t}\right\|_{L^{2}}^{2}+\left\|u_{t}\right\|_{L^{4 / \alpha}}^{2}\right) d t+C \int_{0}^{T^{*}}\left(\left\|\nabla^{2} u_{t}\right\|_{L^{2}}^{2}+1\right) d t \\
& \leq C .
\end{aligned}
$$

It follows from (4.69) and (2.1) that

$$
\sup _{0 \leq t \leq T^{*}} t\|\dot{u}\|_{L^{8 / \alpha}}^{2} \leq C \sup _{0 \leq t \leq T^{*}} t\left\||x|^{\alpha / 4} \nabla \dot{u}\right\|_{L^{2}}^{2} \leq C,
$$

which yields that

$$
\begin{aligned}
\sup _{0 \leq t \leq T^{*}} t\left\|u_{t}\right\|_{L^{8 / \alpha}}^{2} & \leq C \sup _{0 \leq t \leq T^{*}} t\left(\|\dot{u}\|_{L^{8 / \alpha}}^{2}+\|u \cdot \nabla u\|_{L^{8 / \alpha}}^{2}\right) \\
& \leq C+C \sup _{0 \leq t \leq T^{*}}\|u\|_{L^{\infty}}^{2}\|\nabla u\|_{L^{8 / \alpha}}^{2} \\
& \leq C .
\end{aligned}
$$

Choosing $p=8 / \alpha$ in both (4.45) and (4.43), together with (4.42), yields that

$$
\sup _{0 \leq t \leq T^{*}} t\left\|\nabla^{4} u\right\|_{L^{2}}^{2} \leq C .
$$

Hence (4.50) follows from (4.61), (4.70), and (4.71) directly.

Now we prove our main result Theorem 1.1.

Proof of Theorem 1.1. Proof. If $\tilde{\rho}=0$, Theorem 1.1 is an easy consequence of Proposition 4.2 and Lemmas 4.3-4.6. It remains to prove that $(\rho, u)$ becomes a classical solution for positive time, that is, for any $\tau \in\left(0, T^{*}\right]$,

$$
u_{t}, \nabla^{2} u, \nabla \rho, \rho_{t} \in C\left(\left[\tau, T^{*}\right] \times \overline{\mathbb{R}^{2}}\right) .
$$

It follows from (4.42) and (4.50) that for any $\tau \in\left(0, T^{*}\right]$,

$$
\sup _{\tau \leq t \leq T^{*}}\left\|\nabla^{2} u\right\|_{H^{2}} \leq C(\tau)
$$

which, together with (4.42), $\left(\nabla^{2} u\right)_{t} \in L^{2}\left(\mathbb{R}^{2} \times\left(0, T^{*}\right)\right)$, gives that for $p \in(2,+\infty)$,

$$
\nabla^{2} u \in C\left(\left[\tau, T^{*}\right] ; H^{1} \cap W^{1, p}\right) \hookrightarrow C\left(\overline{\mathbb{R}^{2}} \times\left[\tau, T^{*}\right]\right) .
$$

It follows from (4.50) and (4.31) that

$$
\sup _{\tau \leq t \leq T^{*}}\left(\left\|u_{t}\right\|_{L^{8 / \alpha}}+\left\|\nabla u_{t}\right\|_{H^{1}}\right)+\int_{\tau}^{T^{*}}\left\|\left(\nabla u_{t}\right)_{t}\right\|_{L^{2}}^{2} d t \leq C(\tau),
$$


which implies for $p \in(2,+\infty)$,

$$
\nabla u_{t} \in C\left(\left[\tau, T^{*}\right] ; L^{2} \cap L^{p}\right) .
$$

It follows from (4.74) and (4.75) directly that

$$
u_{t} \in C\left(\overline{\mathbb{R}^{2}} \times\left[\tau, T^{*}\right]\right) .
$$

We deduce from (4.30) and (4.42) that

$$
\nabla \rho \in L^{\infty}\left(0, T^{*} ; H^{2}\right), \quad(\nabla \rho)_{t} \in L^{\infty}\left(0, T^{*} ; L^{2}\right),
$$

which yields that for $p \in(2,+\infty)$,

$$
\nabla \rho \in C\left(\left[0, T^{*}\right] ; H^{1} \cap W^{1, p}\right) \hookrightarrow C\left(\overline{\mathbb{R}^{2}} \times\left[0, T^{*}\right]\right) .
$$

It follows from $(1.1)_{1},(4.73)$, and (4.77) that

$$
\rho_{t} \in C\left(\overline{\mathbb{R}^{2}} \times\left[0, T^{*}\right]\right),
$$

which together with (4.73), (4.76), and (4.77) gives (4.72).

If $\tilde{\rho}>0$, after bounding $\|u\|_{L^{2}\left(\mathbb{R}^{2}\right)}$ and $\|\dot{u}\|_{L^{2}\left(\mathbb{R}^{2}\right)}$ by Lemma 2.5 , we obtain Theorem 1.1 as above. Hence, the proof of Theorem 1.1 is complete.

Acknowledgement. Zhen would like to express her sincere thanks to Professor Zhouping Xin for his interests in this work and for stimulating discussions. Zhen would also like to thank Professor Jing Li for some helpful discussions.

\section{REFERENCES}

[1] F. Catrina and Z.Q. Wang, On the Caffarelli-Kohn-Nirenberg inequalities: Sharp constants, existence (and nonexistence), and symmetry of extremal functions, Commun. Pure Appl. Math., 54, 229-258, 2001.

[2] L.A. Caffarelli, R. Kohn and L. Nirenberg, First order interpolation inequalities with weights, Composition Math., 53, 259-275, 1984.

[3] Y. Cho, H.J. Choe, and H. Kim, Unique solvability of the initial boundary value problems for compressible viscous fluids, J. Math. Pures Appl., 83, 243-275, 2004.

[4] Y. Cho and H. Kim, On classical solutions of the compressible Navier-Stokes equations with nonnegative initial densities, Manuscript Math., 120, 91-129, 2006.

[5] Y. Cho and H. Kim, Existence results for viscous polytropic fluids with vacuum, J. Diff. Eqs., $228,377-411,2006$.

[6] H.J. Choe and H. Kim, Strong solutions of the Navier-Stokes equations for isentropic compressible fluids, J. Diff. Eqs., 190, 504-523, 2003.

[7] B. Desjardins, Regularity of weak solutions of the compressible isentropic Navier-Stokes equations, Commun. Part. Diff. Eqs., 22, 977-1008, 1997.

[8] E. Feireisl, Dynamics of Viscous Compressible Fluid, Oxford University Press Inc., 2004.

[9] D. Gilbarg, N. Trudinger, Elliptic Partial Differential Equations of Second Order, 2nd ed., Springer, 1983.

[10] D. Hoff, Global existence for 1D, compressible, isentropic Navier-Stokes equations with large initial data, Trans. Amer. Math. Soc., 303, 169-181, 1987.

[11] D. Hoff, Global solutions of the Navier-Stokes equations for multidimensional compressible flow with discontinuous initial data, J. Diff. Eqs., 120, 215-254, 1995.

[12] D. Hoff, Compressible ow in a half-space with Navier boundary conditions, J. Math. Fluid Mech., 7, 315-338, 2005. 
[13] X.D. Huang, J. Li, Z. Luo, and Z.P. Xin, Global existence and blowup phenomena for smooth solutions to the two-dimensional compressible flows with vacuum, preprint.

[14] X.D. Huang, J. Li, and Z.P. Xin, Global well-posedness of classical solutions with large oscillations and vacuum to the three-dimensional isentropic compressible Navier-Stokes equations, Comm. Pure Appl. Math., accepted.

[15] A.V. Kazhikhov and V. Shelukhin, Unique global solution with respect to time of initialboundary value problems for one-dimensional equations of a viscous gas, J. Appl. Math. Mech., 41, 273-282, 1977.

[16] P.L. Lions, Mathematical Topics in Fluid Mechanics, Volume 2, Compressible Models, Oxford Science Publication, Oxford, 1998.

[17] O.A. Ladyzenskaja, V.A. Solonnikov, and N.N. Ural'ceva, Linear and Quasilinear Equations of Parabolic Type, American Mathematical Society, Providence, RI, 1968.

[18] A. Matsumura and T. Nishida, The initial value problem for the equations of motion of viscous and heat-conductive gases, J. Math. Kyoto Univ., 20, 67-104, 1980.

[19] J. Nash, Le problème de Cauchy pour les équations différentielles d'un fluide général, Bull. Soc. Math. France., 90, 487-497, 1962.

[20] R. Salvi and I. Straškraba, Global existence for viscous compressible fluids and their behavior as $t \rightarrow \infty$, J. Fac. Sci. Univ. Tokyo Sect. IA, Math., 40, 17-51, 1993.

[21] D. Serre, Solutions faibles globla des équations de Navier-Stokes pour un fluide compressible, C.R. Acad. Sci. Paris Sér. I Math., 303, 639-642, 1986.

[22] D. Serre, Sur l'équation monodimensionnelle d'un fluide visqueux, compressible et conducteur de chaleur, C.R. Acad. Sci. Paris Sér. I Math., 303, 703-706, 1986.

[23] J. Serrin, On the uniqueness of compressible fluid motion, Arch. Rational Mech. Anal., 3, 271-288, 1959.

[24] J. Simon, Compact sets in the space $L^{p}(0, T ; B)$, Ann. Mat. Pura Appl., 146, 65-96, 1987. 